\title{
The Density Matrix Renormalization Group for finite Fermi systems
}

\author{
Jorge Dukelsky† and Stuart Pittel\$ $\oint$ \\ $\dagger$ Instituto de Estructura de la Materia, Consejo Superior de \\ Investigaciones Cientificas, Serrano 123, 28006 Madrid, Spain \\ $\ddagger$ Bartol Research Institute, University of Delaware, Newark, \\ Delaware 19716, USA
}

\begin{abstract}
The Density Matrix Renormalization Group (DMRG) was introduced by Steven White in 1992 as a method for accurately describing the properties of onedimensional quantum lattices. The method, as originally introduced, was based on the iterative inclusion of sites on a real-space lattice. Based on its enormous success in that domain, it was subsequently proposed that the DMRG could be modified for use on finite Fermi systems, through the replacement of real-space lattice sites by an appropriately ordered set of single-particle levels. Since then, there has been an enormous amount of work on the subject, ranging from efforts to clarify the optimal means of implementing the algorithm to extensive applications in a variety of fields. In this article, we review these recent developments. Following a description of the realspace DMRG method, we discuss the key steps that were undertaken to modify it for use on finite Fermi systems and then describe its applications to Quantum Chemistry, ultrasmall superconducting grains, finite nuclei and two-dimensional electron systems. We also describe a recent development which permits symmetries to be taken into account consistently throughout the DMRG algorithm. We close with an outlook for future applications of the method.
\end{abstract}

Submitted to: Rep. Prog. Phys.

PACS numbers: 05.10.Cc, 21.60.Cs, 31.15.Ar, 71.10.Fd, 75.10.Jm

$\S$ To whom correspondence should be addressed (pittel@bartol.udel.edu) 


\section{Introduction}

The properties of non-relativistic many-body quantum systems, as arise in many branches of physics, are described by the Schrödinger equation. Unfortunately, the Schrödinger equation can be solved exactly in but a few exceptional cases, either involving one-dimensional or symmetry-restricted Hamiltonians or for systems with very few particles. For almost all problems, reliable approximate solutions must be sought. Many methods of approximately solving the Schrödinger equation for manybody quantum systems have been developed, often tailored to the specific system or problem under investigation. Especially challenging has been to come up with methods that can treat large numbers of particles with great accuracy, particularly in the vicinity of a phase transition. A major breakthrough was the development of the numerical renormalization group (RG) method by Wilson [1] in the mid-1980s, which provided for the first time an efficient strategy for solving the Kondo problem [2. The enormous power of the RG method immediately suggested its application to quantum lattices. It soon became clear, however, that the Wilson RG method ran into difficulties when dealing with such systems.

The Density Matrix Renormalization Group (DMRG) was introduced by White in 1992 [3, 4, 5] in an effort to overcome the limitations of Wilson's RG in describing one-dimensional (1D) quantum lattice models. The new method was soon shown to be extremely powerful, producing results for the ground state energy of the $\mathrm{S}=1$ Heisenberg chain that were accurate to twelve significant figures [4, well beyond the precision of large-scale diagonalization methods combined with finite size corrections or of Monte Carlo techniques.

The DMRG is an approximate variational procedure that is rooted in Wilson's onion picture [6]. In the context of quantum lattices, the idea is to start with a set of lattice sites and then to iteratively add to it subsequent sites until all have been treated. At each iteration, the dimension of the enlarged space increases as the product of the dimension of the initial subspace and that of the added site. The renormalization group procedure consists of reducing the enlarged space to the same dimension as the initial subspace and then transforming all operators to this new truncated basis (renormalization).

One of the key features that distinguishes the DMRG from Wilson's RG is the criterion by which the truncation is implemented. While the Wilson RG retains the lowest Hamiltonian eigenstates of the enlarged space, the DMRG uses a very different strategy. Here the idea is to construct the reduced density matrix for the enlarged space in the presence of a medium that approximates the rest of the Hilbert space, then to diagonalize this density matrix, and finally to maintain only those states with the largest density matrix eigenvalues. This method of truncation is guaranteed to be optimal in the sense that it maximizes the overlap of the approximate (truncated) wave function with the wave function prior to truncation.

Following the ideas just described, one can treat the entire set of "onion" layers. Depending on the manner in which correlations between layers fall off and in the 
choice of the order in which layers are treated, this can sometimes lead to an accurate representation of the ground state of the system. Usually, it does not, however, since the early layers know nothing of the physics of those treated subsequently. This suggests the use of a "sweeping" algorithm, whereby once all layers have been sampled, we simply reverse direction and update them based on the information of the previous "sweep". Such a sweeping algorithm can be iteratively implemented until acceptable convergence in the results has been achieved.

The real-space DMRG (rDMRG), as described above, has been applied extensively and with enormous success to many 1D systems [5, 7], such as spin chains and ladders [8, 9, t-J [10] and Hubbard models [11, models with disorder [12, models with impurities [13], polymers [14, and the Kondo model [15]. Extension of the method to 2D lattices [16] requires establishing a path that covers all of the sites of the lattice. Since there are many possible paths through a 2D lattice, it is critical that the path chosen conform to the dominant correlations of the problem. Electrons in small square or rectangular lattices with t-J or Hubbard Hamiltonians [17] have been treated using this DMRG strategy, though with much less precision than for 1D systems. Problems in 3D are beyond the scope of the DMRG in real space.

A pedagogical introduction to the real space DMRG method together with a summary of some of its most important applications can be found in the proceedings of the workshop "Density-Matrix Renormalization" [18] held in Dresden in September 1998.

Since then, there have been many important developments and advances of the DMRG, which has opened up the possibility of its application to finite Fermi systems. These new developments originated with the introduction of a transformation of the DMRG from real space to momentum space (kDMRG). The kDMRG method was first discussed by Xiang [19] and applied to the Hubbard model in 2D. The results were not very encouraging, however, and the method was therefore not pursued at that time for other 2D quantum lattices.

This method was recovered in a slightly modified form in 1999 in an effort to build a DMRG methodology appropriate to problems in quantum chemistry [20, 21]. In this approach, the momentum basis was replaced by a basis of single-particle orbitals, so as to better accommodate the dynamics of the problem. The first applications were reported in refs. [20, 21]. Several other applications to problems in quantum chemistry were subsequently reported [22, 23].

A somewhat different extension of the DMRG methodology was proposed in refs. 24, 25], in the context of a study of ultrasmall superconducting grains. In that work, a modification of the algorithm - called the particle-hole DMRG (phDMRG) - was proposed in order to optimally account for the crucial correlations close to the Fermi energy. The method was shown to work extremely well for superconducting grains up to very large dimensions. The phDMRG was then introduced for possible application in large-scale nuclear shell-model calculations [26, 27, 28].

Several other important applications of the extended methodology have also been 
reported recently, including applications to electrons in the lowest three Landau levels [29, 30, 31, 32, 33.

The DMRG methodology, both in real space and in the versions appropriate to finite Fermi systems, usually violates some symmetries of the underlying Hamiltonian. While this is not critical when studying certain features of the associated quantum systems, it may be critical when dealing with others. There have been some recent efforts to modify the DMRG algorithm [34, 35, 36, 37] to permit the preservation of symmetries throughout the iterative truncation process.

In this review, we summarize the rapid progress now being made on the DMRG methodology and the wide variety of important applications that have been reported recently for strongly correlated finite Fermi systems. We begin in Section II with an historical overview of the method and review its application in real space to quantum lattices. In the same section, we describe the basic features of the $\mathrm{kDMRG}$ extension and discuss its application to quantum lattices in more than one dimension. In Section III, we begin our focus on finite Fermi systems, discussing the modifications to the general DMRG algorithm needed in such cases and reviewing the applications to date. Then in Section IV we discuss the issue of symmetry restoration, summarizing recent developments and describing in some detail how this new machinery can be used in the context of angular momentum restoration. In Section V, we close the presentation by providing some perspectives for future applications of the DMRG approach in finite Fermi systems.

\section{Historical overview of the DMRG method}

\subsection{The many-body quantum problem}

A quantum many-body problem is completely defined by giving a basis of one-body states, a Hamiltonian operator and the number of particles. Usually, the one-body states are obtained from a self-consistent mean-field calculation, such as Hartree-Fock (HF), or by some optimized orbital basis. In atomic and molecular physics [38], for example, the electrons move in the attractive Coulomb field generated by the atomic nucleus. The residual electron-electron interaction can be taken into account in mean field to give a set single-electron energy states, which then can serve as the starting basis. In nuclear physics [39, the nucleon-nucleon mean field generates a set of selfconsistent single-nucleon levels, which can provide the basis. Alternatively, we can ignore detailed self-consistency and choose a basis for convenience. Convenient singlenucleon bases used frequently in nuclear physics include the eigenstates of a harmonic oscillator potential (all localized) and the eigenstates of a Woods-Saxon potential (both localized and continuum states).

Though the main goal of this review is to present the latest developments using the DMRG method in such finite Fermi systems, we will begin with an historical overview, starting with the development of the method for quantum lattices and its applications in 
that domain. Here, the single-particle basis state is replaced by a single active electron level, located at a given site in real space.

One of the most well-known models of strongly interacting electrons on a lattice is the Hubbard model [40]. It describes the hopping of electrons between nearest neighbor sites (atoms) subject to a repulsive Coulomb interaction between them when they occupy the same site. The Hamiltonian of the Hubbard model takes the form

$$
H=-t \sum_{<i j>\sigma}\left(c_{i \sigma}^{\dagger} c_{j \sigma}+c_{j \sigma}^{\dagger} c_{i \sigma}\right)+U \sum_{i} n_{i \uparrow} n_{i \downarrow}
$$

The operator $c_{i \sigma}^{\dagger}\left(c_{i \sigma}\right)$ creates (annihilates) an electron on site $i$ with spin projection $\sigma$. The first term represents the hopping of electrons with intensity $t$. The strength of the on-site Coulomb repulsion is $U$ and $n_{i \sigma}=c_{i \sigma}^{\dagger} c_{i \sigma}$ denotes the number operator for an electron on site $i$ with spin projection $\sigma$.

Assuming that we have a system with $N$ sites, the exact solution of the problem amounts to constructing the many-body basis of states in the appropriate Hilbert space, calculating the matrix elements of the Hamiltonian in this basis and then diagonalizing. In the Hubbard model, each site admits precisely four states, as it can be empty, it can contain an electron with spin up, it can contain an electron with spin down, or it can be fully occupied by two electrons. Thus, the dimension of the Fock space is naively $4^{N}$, growing exponentially with the number of sites. Because of conservation of symmetries in the many-body basis, e.g. total particle number, total spin, total momentum, etc., the Hamiltonian matrix separates into blocks, each with reduced dimension. Nevertheless, the order of magnitude of each block dimension is still comparable to the full Fock space dimension and thus likewise grows exponentially with the number of lattice sites. For this reason, exact large-scale diagonalization using the Lanczos or Davidson algorithms is only feasible for a small number of sites. The largest reported exact diagonalization of the Hubbard model, for example, was for a $4 \times 4$ 2D lattice.

Exact diagonalization, combined with finite size extrapolation, has proven very useful when trying to understand the gross features of several lattice models. Nevertheless, we would like to do better, by being able to treat larger systems. Obviously, if we want to treat larger systems we have to implement an efficient truncation strategy, one that permits an accurate description of the low-energy properties of the system while only keeping a reasonably small part of the full Hilbert space.

In the finite Fermi systems that arise in Quantum Chemistry (QC) and in Nuclear Structure (NS), similar issues arise as regards the size of the Hilbert space and the need for an efficient truncation strategy. In those fields, a variety of methods have been developed and used with great success in the study of small systems, such as simple molecules and light nuclei. In QC, for example, the Configuration Interaction (CI) method [38] working in a HF basis with a limited set of particle-hole states can be used to build a truncated many-body Hilbert space in which to diagonalize the Hamiltonian and obtain approximate solutions of the Schrödinger equation. In NS, the Shell Model (SM) [41] starts with a nuclear mean field generated by the average 
interaction of one nucleon with all the others. For simplicity, the mean field is often modelled by a harmonic oscillator potential with a spin-orbit coupling, defining a set of shells well separated in energy. The nuclear interaction expressed in this basis is typically diagonalized for a given nucleus within one major shell for each type of nucleon. Despite the enormous truncation inherent in both the CI approach in QC and the SM approach in NS, the methods are limited as to the systems that can be treated accurately. In NS, for example, exact shell-model diagonalization techniques cannot be applied to nuclei beyond the $1 f-2 p$ shell, leaving outside the scope of detailed shell-model microscopy most medium-mass and heavy nuclei.

Going beyond the limits of exact diagonalization techniques, even within the restricted bases just described, requires a new kind of truncation strategy. One that has been used with great success in many systems is the Monte Carlo approach. Another is the renormalization group ( $R G)$ approach [1, the subject of this review. In this method, a set of renormalization transformations that gradually grow the size of the system while keeping the dimension of the configuration space constant is introduced. The procedure is implemented iteratively. At each step, the system is grown by the addition of new degrees of freedom. The enlarged system is then truncated to the same size as before, and a renormalization is implemented which transforms the Hamiltonian and all other relevant operators to the truncated space. The numerical renormalization group of Wilson represented a breakthrough in the application of RG ideas to a nontrivial strongly correlated system, the single-impurity Kondo problem [2]. Later on it became clear that the Wilson RG (WRG) could not be applied to other strongly correlated lattice problems [42, for reasons that we will discuss below. Nevertheless, the method and its failure provide the basis for the development of the DMRG.

\subsection{The Wilson Renormalization Group}

In this subsection, we briefly describe the numerical renormalization group of Wilson for a generic one-dimensional lattice. While we will have in mind for concreteness the Hubbard Hamiltonian (II), the formalism we will describe is general enough to incorporate other one-dimensional lattice models, including the Kondo problem. In fact, Wilson first formulated his numerical RG after mapping the Kondo problem onto a 1D lattice in energy space.

The starting point of a WRG calculation (and as we will discuss later the DMRG as well) is a small system for which we know all of the states and for which we can calculate the matrix elements of all relevant operators including the Hamiltonian. We begin, therefore, by considering a set of $L$ contiguous lattice sites - which we will call a block - with $L$ small enough so that we can exactly diagonalize the Hamiltonian in the associated subspace. Figure 1 illustrates a chain of lattice sites, with the filled dots representing the sites of the initial system - $L=2$ in this case - and the open dots representing all other sites in the problem. For $L=2$, the number of states in the block for the Hubbard model is $m=16$. 


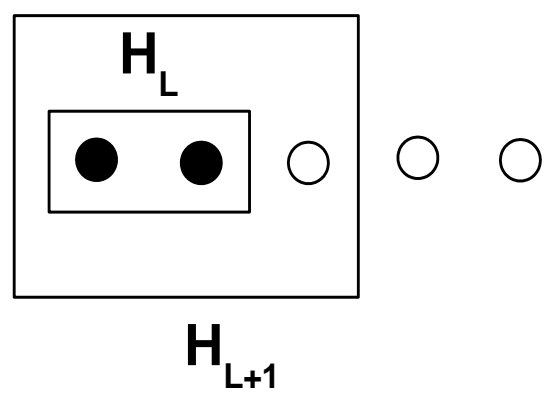

Figure 1. Schematic illustration of the sites on a one-dimensional lattice and the Wilson Renormalization Group method of enlarging blocks of sites. The sites denoted by a bullet $\bullet$ have already been treated, and the next site to the right is in the process of being added.

The WRG procedure begins with a calculation of the matrix elements of all relevant operators in the initial block. The relevant operators are those that enter

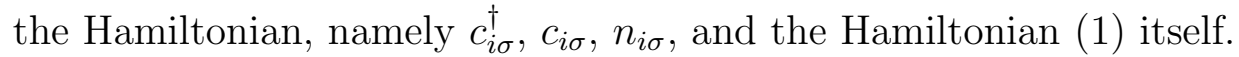

Then we continue as follows:

1. Add the site $L+1$ and calculate the matrix elements of all operators in the enlarged block space of $4 m$ states. [Note: If we wish, we can partition the states of a site and add only 2 states, rather than 4 in each step. When this is done, the enlarged block space has $2 m$ states instead of $4 m$.]

2. Diagonalize the Hamiltonian and select the lowest $m$ eigenvalues and the corresponding eigenvectors.

3. Transform all operators in the enlarged block to a truncated basis involving these $m$ states only.

4. If $L+1$ is lower that the total number of sites $N$, substitute $L+1$ for $L$ and repeat steps $\mathbf{1} \rightarrow \mathbf{3}$.

The key ingredient in the Wilson renormalization strategy is step 2, whereby $m$ states are selected from the $4 m$ (or $2 m$ ) states of the enlarged block by a purely energetic criterion. Clearly, the accuracy of the method relies on the assumption that the lowenergy properties of the system are fully contained in the low-energy states of the individual blocks into which the system was partitioned. Indeed, this was the case for the Kondo problem, which was solved by Wilson by transforming it to a discretized energy basis, and for other impurity models like the Anderson model [43].

The Kondo model describes the antiferromagnetic interaction of the conduction electrons with a localized impurity. Its Hamiltonian in a discretized energy basis takes the form

$$
H=\frac{1}{2}\left(1+\Lambda^{-1}\right) \sum_{n=0}^{\infty} \Lambda^{-n / 2}\left(f_{n \mu}^{\dagger} f_{n+1 \mu}+f_{n+1 \mu}^{\dagger} f_{n \mu}\right)+2 J \rho f_{0 \mu}^{\dagger} \sigma_{\mu \nu} f_{0 \nu},
$$

where $f_{n \mu}^{\dagger}\left(f_{n \mu}\right)$ creates (annihilates) an electron in the energy interval $n$ with spin $\mu$, $J$ is the strength of the antiferromagnetic interaction between the conduction electrons 


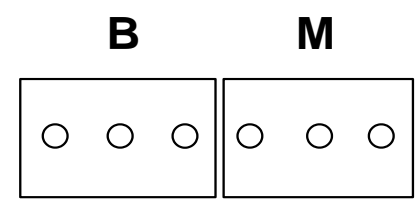

Figure 2. Schematic illustration of the two-block structure of the Density Matrix Renormalization Group.

and the impurity, $\rho$ is the density of states and $\Lambda$ is a discretization parameter defining the intervals of energy. For $\Lambda>1$ and beginning from the origin $(n=0)$ of the linear chain, the interaction along the chain decays exponentially, assuring the validity of the truncation procedure. The WRG was the first numerical implementation of the RG to a non-perturbative problem like the Kondo model, for which it had enormous success. As such, it is still used to this day to study problems with one or few impurities.

Unfortunately, the Wilson RG cannot be applied to other lattice problems. For example, when it is applied to 1D Hubbard models it begins to deviate significantly from the exact results in the fourth iteration. Obviously, the problem resides in the fact that the truncation strategy was based solely on energy arguments. The solution to this problem was proposed by White with his introduction of the DMRG, which we turn to in the next subsection.

\subsection{The Density Matrix Truncation Strategy}

The essential limitation of the WRG, as discussed in the previous subsection, is the method used for truncation at each RG iteration step. Once the enlarged block is constructed by adding the site $L+1$ in step 1, the Hamiltonian in the $L+1$ - site subspace of the total Hilbert space is diagonalized, without considering the coupling to the rest of the space. Obviously, the method will work well when this coupling is perturbatively small. This is the case for Hamiltonians that can be transformed to the form (2). In general, however, the WRG performs very poorly for strongly correlated lattice systems. In searching for a more suitable method of truncation, White analyzed the case of a block immersed in a medium representing the rest of the system, as depicted in figure 2

Assume that we know the ground state $|\Psi\rangle$ of the system and the complete set of $p$ states of the block $B$ and $t$ states of the medium $M$. Then the ground state of the full system $|\Psi\rangle$ can be expanded in the product space of the block and the medium as

$$
|\Psi\rangle=\sum_{i=1, p} \sum_{j=1, t} \Psi_{i j}|i\rangle_{B}|j\rangle_{M} .
$$

Our aim is to determine the best approximation $\left|\Psi^{\prime}\right\rangle$ to the ground state $|\Psi\rangle$ when we select only $m$ states of the block, with $m<p$. To show how this is done, we will make use of the concept of the singular value decomposition (SVD) of a matrix [44]. 
The singular value decomposition of the wave function matrix $\Psi_{i j}$ in (3) permits its factorization as

$$
\Psi_{i j}=\sum_{\alpha=1, p} a_{\alpha} u_{i \alpha} v_{\alpha j}
$$

where the $a_{\alpha}$ are real positive numbers greater than or equal to $0, u$ is an orthogonal square matrix of dimension $p$ and $v$ is a column orthogonal rectangular matrix of dimension $p \times t$. The coefficients $a_{\alpha}$ are known as the weight factors of the SVD.

Up to this point, everything is exact. Now consider what happens if some of the weight factors in (44) are zero or very small. If that is the case, then $\Psi$ can be very well approximated by discarding them, i.e. by truncating the sum in (4) to those with the largest weights. If fact, it can be shown that the approximate wave function factorized as in (41) but retaining the largest $m$ weight factors has the largest overlap with the exact wave function for any factorization involving $m$ terms.

In what follows, we will use the singular value decomposition as a convenient factorization for deriving the conditions for optimal truncation in a density matrix formalism. It is interesting to note, however, that the same approach has been used to develop a powerful new technique for numerically diagonalizing the nuclear shellmodel problem without actually introducing the DMRG 45].

Now consider the reduced density matrix of the block associated with the ground state wave function (3), which is defined as

$$
\rho_{i j}^{B}=\sum_{k=1, t} \Psi_{i k} \Psi_{j k}
$$

with the summation running over the $t$ states of the medium. Inserting the factorization (41) into (5) and using the orthogonality relations of the matrices $u$ and $v$ and the normalization of the wave function, we can express the reduced density matrix (5) as

$$
\rho_{i j}^{B}=\sum_{\alpha=1, p} a_{\alpha}^{2} u_{i \alpha} u_{\alpha j}
$$

From this we see that $u$ is the matrix of eigenvectors of the reduced density matrix and $\omega_{\alpha}=a_{\alpha}^{2}$ are the corresponding eigenvalues. Each eigenvalue $\omega_{\alpha}$ represents the probability of finding the block state $u_{\alpha}$ in the ground state $|\Psi\rangle$ of the coupled system. The normalization of the wave function $\Psi$ guarantees that the trace of $\rho^{B}$ is 1 . Clearly a truncation to $m$ states implies the selection of the largest $m$ eigenvalues and corresponding eigenvectors. The discarded $p-m$ states contribute with a probability $1-P_{m}=1-\sum_{\alpha=m+1}^{p} \omega_{\alpha}$. This parameter provides an important control on the accuracy of the truncation.

Up to now, we have considered the system as being in a pure state. A similar analysis can be carried out for systems in a mixed state. Mixed states naturally arise when considering a system at finite temperature $(T)$. They can even arise at $T=0$ if we decide to target several states with the DMRG truncation strategy. By using mixed density matrices associated with several states, we can build a truncation 
algorithm whereby the gradually enlarging block optimally reflects the physics of all of the associated states and not just the physics of the ground state. The most important states of the block, when it is immersed in a mixed-state system, are those corresponding to the largest eigenvalues of the mixed density matrix,

$$
\rho_{i j}^{B}=\sum_{a} W_{a} \sum_{k=1, t} \Psi_{i k}^{a} \Psi_{j k}^{a},
$$

where $W_{a}$ is the probability with which a given pure state $\left|\Psi^{a}\right\rangle$ enters in the mixed state. In the case of systems at finite temperature, $W_{a}$ is the Boltzmann factor. For a mixed state at $\mathrm{T}=0$, we have the freedom to select the weights so as to assign a relative importance to each state.

\subsection{The DMRG Method for Quantum Lattices: Real space}

In the previous subsections we described how we can choose the optimal states of a part of a quantum system from its density matrix in the presence of a medium approximating the rest of the system. Here, we show how this can be used in a systematic treatment of many-site quantum lattices.

We begin by verbally detailing the key ingredients of the method. The basic idea is to systematically take into account the physics of all sites on the lattice. The procedure begins by starting at the left (or right) edge of the lattice and then gradually growing the size of the space that has been taking into account by adding the other lattice

sites. At each step of this iterative procedure, a truncation is implemented, so as to optimally take into account the effect of the most important states within that part of the problem. The calculation is carried out as a function of the number of states that are maintained after each iteration. This parameter, which we call $m$, is gradually increased and the results are plotted against it. Experience suggests that the results converge exponentially with $m$. Thus, once we achieve changes with increasing $m$ that are acceptably small we terminate the calculation.

In the following, we show how this strategy is implemented at two different levels of complexity, referred to as the infinite algorithm and the finite algorithm, respectively. We then describe some technical details of importance to the computational feasibility of applying the method.

2.4.1. The Infinite Algorithm. The infinite algorithm, as we will soon see, can only be applied meaningfully in the presence of a uniform (or translationally invariant) lattice. Thus, in what follows we assume that the lattice under discussion satisfies this criterion.

Consider such a lattice, as illustrated in figure 3 . Assume that each site on the lattice admits $l$ states. In the case of a spin- $1 / 2$ chain, $l=2$, corresponding to spin up or spin down. In the case of the t-J model, $l=3$, as the site can also be empty. Finally, as noted earlier, in the Hubbard model a site can also have both spin-up and spin-down particles and $l=4$. 


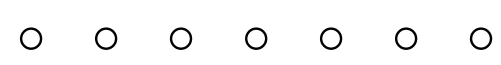

(a)

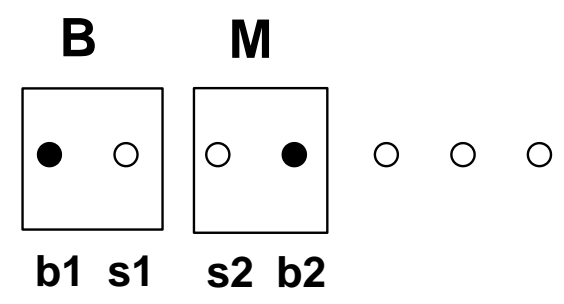

(b)

Figure 3. Schematic illustration of the infinite DMRG algorithm. (a) The sites on a uniform one-dimensional lattice that will be used in subsequent discussion of the DMRG algorithm. (b) The two-block structure of the algorithm. A bullet $(\bullet)$ refers to a block of sites and a circle (o) to a single site. $B$ refers to the enlarged block, which is comprised of $b 1$ and $s 1$. $M$ refers to the enlarged medium, which is comprised of $s 2$ and $b 2$.

We now begin to add sites, by assumption from the left. Until we have included enough sites $k$ so that $l^{k}>m$, no truncation is required. We thus begin our discussion after we have added a sufficient number of sites to be in the truncation regime. We assume that we have already treated the leftmost $k$ lattice sites and have identified the most important $m$ states.

At that point, we can view the problem as illustrated in figure $3 \mathrm{~b}$. The collection of $k$ sites that have already been treated is represented by a $\bullet$ and denoted $b 1$. Immediately to its right is another site, which contains $l$ states and is denoted $s 1$. We then have another site to its right, which also contains $l$ states and is denoted $s 2$. After that we have another block, representing the optimal states from sites $k+3 \rightarrow 2 k+2$. Because of the translational invariance of a uniform quantum lattice, all parts of the lattice look the same. As a consequence, once we know the optimal states from sites $1 \rightarrow k$ we also know the optimal states from any other group of $k$ contiguous sites. This second block is denoted $b 2$. And then finally we have other sites to the right that are still to be considered.

We then consider as an enlarged block the $m \times l$ states obtained by adding the states of site $s 1$ to those of block $b 1$. We will use the Density Matrix strategy described in the previous subsection to truncate from these $m \times l$ states to the optimal $m$ states, the same number that we had before we enlarged the block. As the medium in which to implement the truncation, we use the set of $m \times l$ states of the site $s 2$ coupled to the block $b 2$. The rationale for growing both the block and the medium at the same time is discussed in ref. 4 . 
Following implementation of the density matrix truncation, we make the following replacements:

- The set of $m$ states of the enlarged block now becomes block $b 1$.

- The next available site to its right becomes $s 1$

- The next site after that becomes $s 2$.

- The truncated set of $m$ states from the next $k+1$ sites becomes block $b 2$.

This leaves us in the same position as in figure $3 \mathrm{~b}$, with a block of $m$ states, followed by a site of $l$ states, then another site of $l$ states and finally a block of $m$ states. As before, we build enlarged blocks out of the first two and out of the second two, respectively. We then truncate the enlarged block from $b 1$ and $s 1$ to the same $m$ states as before. And we then use the translational symmetry of the problem to form another set of four objects, $b 1, s 1, s 2, b 2$, and continue the iteration. This iterative process can be continued until the block includes half of the sites. At this point, the superblock contains the physics of all of the sites and thus gives a representation of the entire problem.

Such a procedure, in which we grow the system from left to right (or from right to left) until all sites have been treated is called the infinite DMRG algorithm. Clearly it requires that the lattice be uniform, so that we can use the translational invariance of the problem to relate the collective structure of the medium to that of the block. In such cases, the accuracy of the procedure still depends on how rapidly the correlations between sites fall off. Exact numerical results can be obtained when the correlations are predominantly between fairly close neighbors or, more precisely, when the correlation length is finite. A typical example is the $S=1$ Heisenberg chain [5].

2.4.2. The Finite Algorithm and Sweeping. Often the above criteria for success of the infinite algorithm are not satisfied and it is necessary to do better. When the lattice is not unform, there is no natural way to build a medium that properly reflects correlations in that part of the lattice. When the correlations do not fall off significantly rapidly, there is no way to take into account the effects of distant sites - those that are not part of the medium - in defining the collective structure of the block. A way to overcome these limitations is to use the so-called finite algorithm, which we now describe.

The basic idea of the finite algorithm is that we sweep forwards and backwards through the set of sites, at each stage updating the information from the previous sweep. We will continue to focus here on quantum lattices, and in that context show how the method is implemented. We will first carry out the discussion for a uniform lattice and then mention the modifications needed when the lattice is not uniform.

As before we begin by growing the system from the left. For a unform lattice, as depicted in fig. 3a, we follow the infinite algorithm until half of the sites have been treated in the left block and half in the right block. In each iteration we store on hard disk all relevant matrix elements within the block for later use. From this point on, the superblocks will involve all the $N$ sites of the finite lattice. We then continue to 


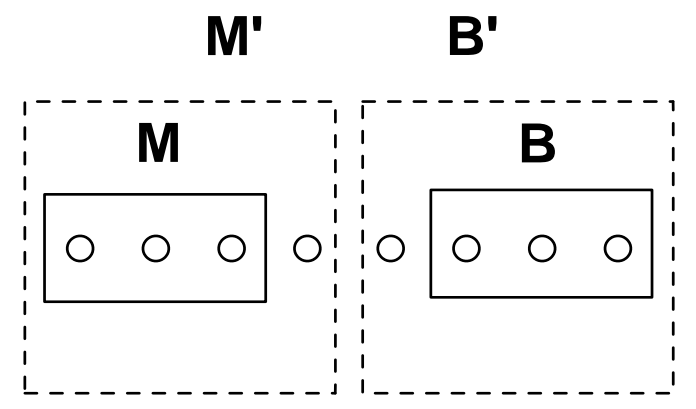

Figure 4. Schematic illustration of the finite DMRG algorithm. B denoted the initial block and $\mathbf{B}^{\prime}$ the enlarged block; $\mathbf{M}$ denotes the initial medium and $\mathbf{M}^{\prime}$ the enlarged medium.

grow the sites in the left block, one at a time. For each enlarged block, we use for the medium the remaining set of sites from the right half. All information on that set of sites is obtained by a reflection from the stored information of the left block from an earlier iteration, assuming inversion symmetry around the center of the lattice.

We continue this process until the left block has been grown to include all sites but three. At this point, we have stored information on all subgroups of sites, from 2 through $N-3$. That information includes the matrix elements of all sub-operators of the Hamiltonian, within the space of states being maintained.

If the lattice is not uniform, we must use a slightly different strategy, since there is no simple prescription for relating the states of the medium to those of the block. One possibility is to use the Wilson RG procedure, growing the system one site at a time and truncating by energy considerations [19. Alternatively, one can use the infinite algorithm, beginning with blocks at the two ends and gradually expanding them by adding sites in between in the direction of the lattice center [15]. In either case, we continue until all sites have been treated and information on all subgroups has been calculated and stored.

At this point, whether dealing with uniform or non-uniform lattices, we reverse the process and sweep from the right, as illustrated in figure 4. We begin to enlarge the right block, by adding one site at a time. The medium for this enlarged block is the set of sites on the left, which consists of the block $M$ and the extra site. Note that all relevant information on the block $M$ was stored in the previous sweep.

At this point, we reverse again, sweeping from left to right. The sweep process is continued iteratively until the results from one sweep are acceptably close to those from the previous sweep. The comparison can be done for the ground-state energy and for the density matrix eigenvalues of the retained states.

Note that the first (or warm-up) stage, in which we sweep through the sites for the first time, need not necessarily produce very good results, since we still have the opportunity to improve on them in subsequent iterations. It is for this reason that the finite algorithm can provide a good approximation to the physics of non-unform lattices. 


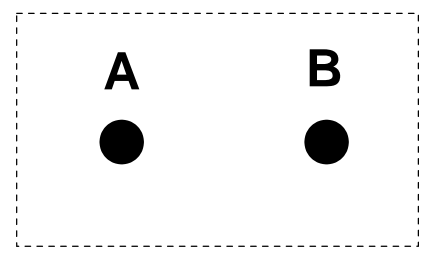

Figure 5. Schematic illustration of block enlargement in the DMRG. A denotes the initial block and $\mathbf{B}$ denotes the block (or site) that is going to be added to it.

2.4.3. Some technical details. For the purposes of this discussion, we assume that the Hamiltonian describing the system under investigation contains one- and two-body terms only and can be written as

$$
H=\sum_{m} \varepsilon_{m} a_{k}^{\dagger} a_{k}+\frac{1}{4} \sum_{m n o p} V_{\text {mnop }} a_{m}^{\dagger} a_{n}^{\dagger} a_{p} a_{o} .
$$

The above Hamiltonian is itself built up out of other operators, which we referred to earlier and will continue to refer to as the Hamiltonian sub-operators. They are

$$
a_{m}^{\dagger}, a_{m}^{\dagger} a_{n}, a_{m}^{\dagger} a_{n}^{\dagger}, a_{m}^{\dagger} a_{n}^{\dagger} a_{p}, a_{m}^{\dagger} a_{n}^{\dagger} a_{p} a_{o},
$$

plus their hermitean adjoints.

A key to the computational feasibility of the DMRG algorithms described earlier in this subsection is that it can be readily automated, using information from previous steps. Here we discuss this point in somewhat more detail.

As we have seen, the DMRG approach involves a systematic enlargement of blocks, followed by a truncation to the same block size as before the enlargement. Consider a representative block enlargement as illustrated in figure [5. The original block $A$ with dimension $d_{A}$ was obtained from an earlier step, at which point the matrix elements of all sub-operators of the Hamiltonian (see Eq. (9)) were calculated and stored. We add to it another block $B$ of dimension $d_{B}$, which can either be a block obtained in an earlier step or a single lattice site. If it is a block obtained from an earlier step, the matrix elements of all sub-operators of the Hamiltonian were calculated and stored at that time for the corresponding set of states. If it is a single lattice site, the matrix elements of the Hamiltonian sub-operators were stored at the outset of the calculation. In either case, the matrix elements of all Hamiltonian sub-operators are accessible in storage when the enlargement of the block is implemented.

At this point, we wish to calculate the matrix elements of the Hamiltonian suboperators in the enlarged block, i.e. in a space of product states

$$
|i, j\rangle=|i\rangle_{A}|j\rangle_{B},
$$

where $i=1, \ldots, d_{A}$ and $j=1, \ldots, d_{B}$.

Consider as a specific example the matrix elements of the one-body operator $a_{m}^{\dagger} a_{n}$ in this basis. They can be obtained as a sum over terms involving products of matrix elements in space $A$ and space $B$, viz: 


$$
\begin{aligned}
\left\langle i, j\left|a_{m}^{\dagger} a_{n}\right| k, l\right\rangle & ={ }_{A}\left\langle i\left|a_{m}^{\dagger} a_{n}\right| k\right\rangle_{A} \delta_{j, l} \\
& +{ }_{B}\left\langle j\left|a_{m}^{\dagger} a_{n}\right| l\right\rangle_{B} \delta_{i, k} \\
& +(-)^{n_{k_{A}}}{ }_{A}\left\langle i\left|a_{m}^{\dagger}\right| k\right\rangle_{A}\left\langle j\left|a_{n}\right| l\right\rangle_{B} \\
& -(-)^{n_{k_{A}}}{ }_{A}\left\langle i\left|a_{n}\right| k\right\rangle_{A}\left\langle j\left|a_{m}^{\dagger}\right| l\right\rangle_{B},
\end{aligned}
$$

where $n_{k_{A}}$ is the number of particles in state $|k\rangle_{A}$. Depending on whether the operator indices refer to the sites in block $A$ or to those in block $B$, a different term in the sum applies. For example, if both $m$ and $n$ refer to sites in block $A$, it is the first term that applies. Since all of the matrix elements in Eq. (11) were calculated and stored earlier, it is straightforward to compute the matrix elements in the enlarged block.

While we have focused for specificity on the one-body particle-hole operator $a_{m}^{\dagger} a_{n}$, it should be clear that the same remarks apply to all of the operators in Eq. (9). Obviously, the number of terms in the sum increases with the number of creation and/or annihilation operators that define the composite operator being treated.

Another key step of the DMRG algorithm is renormalization, whereby all suboperators of the Hamiltonian are transformed to the truncated basis of block states emerging from the density matrix diagonalization, viz:

$$
\rho^{B}\left|\alpha>=\omega_{\alpha}^{B}\right| \alpha>
$$

where

$$
\left|\alpha>=\sum_{i} X_{i}^{\alpha}\right| i>
$$

The matrix elements of any Hamiltonian sub-operator $O$ in the $4 m$-dimensional enlarged block space $\mid i>$ can be transformed to the truncated $m$-dimensional block space of states $\mid \alpha>$ according to

$$
O_{\alpha \beta}=\sum_{i j} X_{i}^{\alpha *} X_{j}^{\beta} O_{i j}
$$

and then stored for use in a subsequent step of the algorithm.

\subsection{The DMRG Method for Quantum Lattices: Momentum space}

In the previous two subsections, we reviewed the key steps in implementing the DMRG algorithm for quantum lattices. The method described worked directly in the real space of lattices, growing the system through the addition of sites.

A key to the success of the DMRG algorithm is being able to define an appropriate order in which to add layers of the onion. In the case of lattices, this means an appropriate order in which to add sites. In one dimension, there is a natural order, namely to add sites either from the right or from the left, always adding the next 
available "nearest neighbor" site(s). This is natural since the interactions that couple sites tend to fall off rapidly with increasing distance between them. But what about lattices in more than one dimension? As an example, consider a 2D square lattice. Near any given site there are four "nearest neighbor" sites. What is the correct path through this set of lattice sites to consider?

For such reasons, it may be useful when dealing with lattices in more than one dimension to consider an alternative labelling of the states of the system, one that is more conducive to a natural ordering.

In 1994, Xiang [19] proposed the possibility of carrying out DMRG calculations in momentum space, rather than in real space. In this section, we review the new ideas that emerged from this proposal. As we will see in the next section, this idea provides the inspiration for the algorithms that have since been developed for finite Fermi systems, the principal focus of this review.

To make the ideas most transparent, we will carry out the discussion in terms of the two-dimensional Hubbard model with nearest-neighbor hopping, as was done in the original paper of Xiang. The Hamiltonian for this model was given earlier in Eq. (1).

The operator that creates a particle in Bloch state $\mathbf{k} \sigma$ with spin $\sigma$ and momentum $\mathbf{k}$ and the one that creates a particle with the same spin $\sigma$ but at lattice site $i$ are related according to

$$
c_{\mathbf{k} \sigma}^{\dagger}=\frac{1}{\sqrt{N}} \sum_{i} e^{i \mathbf{k} \cdot \mathbf{r}_{\mathbf{i}}} c_{i \sigma}^{\dagger},
$$

where $N$ is the total number of sites in the lattice and $\mathbf{r}_{\mathbf{i}}$ is the coordinate-space location of site $i$.

Using this, it is straightforward to transform the Hubbard Hamiltonian (11) to momentum space,

$$
H=\sum_{\mathbf{k} \sigma} \epsilon_{\mathbf{k}} \hat{n}_{\mathbf{k} \sigma}+\frac{U}{N} \sum_{\mathbf{p}, \mathbf{k}, \mathbf{q}} c_{\mathbf{p}-\mathbf{q} \uparrow}^{\dagger} c_{\mathbf{k}+\mathbf{q} \downarrow}^{\dagger} c_{\mathbf{k} \downarrow} c_{\mathbf{p} \uparrow},
$$

where

$$
\hat{n}_{\mathbf{k} \sigma}=c_{\mathbf{k} \sigma}^{\dagger} c_{\mathbf{k} \sigma}
$$

is the number operator for particles with spin $\sigma$ and momentum $\mathbf{k}$ and

$$
\epsilon_{\mathbf{k}}=-t \sum_{<i, j>} e^{i \mathbf{k} \cdot\left(\mathbf{r}_{\mathbf{i}}-\mathbf{r}_{\mathbf{j}}\right)}
$$

is the energy dispersion of the particles, which is explicitly diagonal in the momentum basis.

In particular, by diagonalizing the one-body hopping term for a 2D lattice we find that

$$
\begin{aligned}
& \epsilon_{\mathbf{k}} \quad=-2 t\left(\cos k_{x}+\cos k_{y}\right), \\
& \mathbf{k} \quad=\left(2 \pi n_{x} / L, 2 \pi n_{y} / L\right), \\
& n_{x}, n_{y}=-L / 2+1, \ldots, L / 2 ; L=\sqrt{N} .
\end{aligned}
$$


Table 1. Energy dispersion for the 2D $4 \times 4$ Hubbard model.

\begin{tabular}{ccc}
\hline$\epsilon / t$ & Degeneracy & $\left(k_{x}, k_{y}\right)$ \\
\hline-4 & 1 & $(0,0)$ \\
-2 & 4 & $(-\pi / 2,0),(0,-\pi / 2),(\pi / 2,0),(0, \pi / 2)$ \\
0 & 6 & $(-\pi / 2,-\pi / 2),(-\pi / 2, \pi / 2),(\pi / 2,-\pi / 2),(\pi / 2, \pi / 2),(\pi, 0),(0, \pi)$ \\
2 & 4 & $(-\pi / 2, \pi),(\pi / 2, \pi),(\pi,-\pi / 2),(\pi, \pi / 2)$ \\
4 & 1 & $(\pi, \pi)$ \\
\hline
\end{tabular}

In table [ we give the momentum "spectrum" for a 2D $4 \times 4$ lattice. Possible values for $k_{x}$ and $k_{y}$ in this case are $-\pi / 2,0, \pi / 2$, and $\pi$. What is clear is that there is a large degeneracy at half filling. While it is natural to consider filling momentum-space levels in order of increasing energy dispersion, it is clear that there will be a problem with degeneracies (or near degeneracies when more general hopping terms are used). Thus, the rationale we gave above for introducing momentum space - namely to provide a more natural ordering of levels (onion layers) - may not turn out to be warranted. Nevertheless, we will discuss what happens when the methodology is in fact used. Before doing so, however, we will first discuss some issues related to the implementation of the momentum-space DMRG algorithm, which we subsequently refer to as the kDMRG.

2.5.1. The size of the problem. As emphasized by Xiang in his original paper, the two-body interaction appearing in (16) contains of order $N^{3}$ terms, since each of the sums goes over $N$ possible momentum values. Thus, a straightforward application of the DMRG algorithm in momentum space would require iteratively calculating and storing matrix elements of $O\left(N^{3}\right)$ operators, which would obviously be very time and memory consuming for a large number of sites. Xiang figured out a very clever way to group the operators, so as to dramatically reduce the number whose matrix elements need to be calculated and stored. Since an analogous strategy will be required in all extensions of the usual rDMRG method, we will now discuss how this is accomplished.

Consider the following set of operators, operating in a subspace $A$ of the full $2 \mathrm{D}$ Hubbard Hilbert space:

$$
\begin{array}{ll}
a_{0}(p, \sigma)=c_{p \sigma} & (p, \sigma) \in A \\
a_{1}(p, \sigma)=\sum_{q} a_{0}^{\dagger}(q, \sigma) a_{0}(p+q, \sigma) & \\
a_{2}(p)=\sum_{q} a_{0}^{\dagger}(q, \uparrow) a_{0}(p+q, \downarrow) & \\
a_{3}(p, \sigma)=\sum_{q_{1} q_{2}} a_{0}^{\dagger}\left(q_{1},-\sigma\right) a_{0}\left(q_{2},-\sigma\right) a_{0}\left(p+q_{1}-q_{2}, \sigma\right) & (p, \sigma) \notin A \\
a_{4}(p)=\sum_{q} a_{0}(q, \downarrow) a_{0}(p-q, \uparrow) . &
\end{array}
$$


The total number of such composite operators is $6 N$, significantly less than the total number of sub-operators of the full Hubbard Hamiltonian in momentum space.

For notational purposes, we only use the above set of composite operators when referring to block $\mathrm{A}$. When considering another block $\mathrm{B}$, we systematically replace $a_{i}$ by $b_{i}$.

The key point is that all matrix elements needed for the iterative algorithm can be obtained from these composite operators. To see this, consider for example the Hubbard Hamiltonian in a product space $\mathrm{A} \times \mathrm{B}$. It can be written as

$$
\begin{aligned}
H_{\mathrm{A} \times \mathrm{B}}= & H_{A}+H_{B}+\frac{U}{N} \sum_{p}\left\{\sum _ { \sigma ^ { \prime } } \left[\frac{1}{4} a_{1}\left(p, \sigma^{\prime}\right) b_{1}\left(-p, \bar{\sigma}^{\prime}\right)+b_{0}^{\dagger}\left(p, \sigma^{\prime}\right) a_{3}\left(p, \sigma^{\prime}\right)\right.\right. \\
& \left.+(a \leftrightarrow b)]+a_{4}^{\dagger}(p) b_{4}(p)-b_{2}(p) a_{2}^{\dagger}(p)\right\}+ \text { h.c. }
\end{aligned}
$$

Note that in the A space, we only need the set of $a$ operators and correspondingly in the B space we only need the analogous set of $b$ operators.

In more complex spaces, with larger numbers of coupled blocks, the same remarks can be straightforwardly shown to apply.

The above development is specific to the Hubbard Hamiltonian of Eq. (11). However, as we will discuss later, similar considerations are essential to preserve the computational feasibility of the DMRG algorithm in the more general quantum problems that arise in the description of finite Fermi systems.

2.5.2. Translational invariance and momentum conservation. As noted earlier, the real-space DMRG when applied to uniform quantum lattices benefits greatly from the fact that all positions on the lattice look the same. Thus, once a certain portion of the lattice has been treated, the same results can be transferred to any equivalent set of sites.

The momentum-space DMRG loses this simplicity. A given group of quantized momentum levels has no obvious relation to any other group.

Of course the equivalence of a given group of sites on a uniform lattice to any other analogous group was a consequence of the translational invariance of the problem. That symmetry is not lost by transforming to momentum space; it simply shows up elsewhere. In particular, in momentum space the translational invariance of the lattice shows up in terms of momentum conservation. When building the superblock, we need not mix states of different total momentum. This has the advantage of reducing the size of the superblocks in momentum space compared to real space, even for the same assumed block dimension.

In building states of a given total momentum in the superblock, we must maintain all allowed momentum states for the blocks themselves. The resulting density matrix for the block will thus contain different momenta. However, it will be block diagonal in momentum and thus likewise be effectively reduced in dimension relative to the corresponding matrix in real space. 
Similar considerations play a role in applications of the DMRG to finite Fermi systems, where symmetries likewise must be treated carefully. In rotationally-invariant systems, for example, angular momentum is a conserved quantum number. As we will discuss in Section IV, full incorporation of angular momentum symmetry can be built into the formalism, but at an increase in complexity. Minimally, however, when working in a basis of single-particle states with well-defined angular momentum projection, we can build states with conserved m-projection and thereby reduce the size of the superblocks that must be diagonalized. Any additive quantum number associated with an Abelian symmetry can be used in this way to reduce the size of the superblock and to make the reduced density matrices block diagonal.

2.5.3. The warm-up phase of the $k D M R G$ algorithm. In a real-space description of uniform lattices, we can use the equivalence of contiguous sites throughout the lattice to facilitate the introduction of a medium with which to build a DMRG superblock in the warm-up phase. In momentum-space, as noted above, this is no longer the case. On the one hand, this makes it clear that it is not appropriate to use the infinite DMRG algorithm when working in momentum space. But even with the finite algorithm it is necessary to adopt a strategy for implementing the warm-up phase, where the first approximation to the description of the the various size blocks is obtained.

In the work of Xiang and the others who have implemented the kDMRG, the procedure has been to perform truncation in the warm-up phase according to Wilson's numerical RG procedure, namely to truncate to the lowest eigenstates of the enlarged block Hamiltonian. As for a real-space description of non-uniform lattices, the expectation is that subsequent sweeps should correct any deficiencies that might exist in this procedure.

2.5.4. Applications of the $k D M R G$. Having described the key elements of the $\mathrm{kDMRG}$ method, we now discuss some of the applications that have been reported.

We first discuss some of the results reported by Xiang in the paper that first introduced the method [19]. The calculations were carried out for the 1D and 2D Hubbard models, using the standard Hamiltonian of Eq. (11). They were performed as a function of the number of lattice sites $N$ and the ratio $U / t$ of the strength of the on-site Coulomb repulsion to that of the one-body hopping term. In the case of the 1D model, all calculations were done at half filling, whereas the 2D calculations considered different filling fractions. In all cases, the number of states $m$ kept in each truncation was 1000 and comparison with exact results was reported where available.

Applications in 1D showed two important points: (1) that the momentum-space DMRG method is significantly more accurate than the Wilson RG when applied to the 1D Hubbard model, and (2) that the kDMRG results for 1D lattices are less precise than those for real-space DMRG.

The latter conclusion is worthy of further comment. As noted earlier, the accuracy of the DMRG algorithm depends critically on how rapidly the correlations fall off. In 
Table 2. Comparison of the ground state energy calculated for the $4 \times 4$ Hubbard model. The exact results are compared with those obtained using the kDMRG method, the Quantum Monte Carlo (QMC) method and the Stochastic Diagonalization (SQ) method for different numbers of electrons $N$ and different values of $U / t$. From Table II of ref. [19]. Reprinted by permission of the American Physical Society.

\begin{tabular}{cccccc}
\hline$U / t$ & $N$ & Exact & $k D M R G$ & $Q M C$ & $S Q$ \\
\hline 2 & 16 & -18.01757 & -18.012 & & \\
4 & 14 & -15.74459 & -15.673 & & \\
4 & 16 & -13.62185 & -13.571 & -13.6 & -13.59 \\
8 & 16 & -8.263 & -8.48 & & \\
\hline
\end{tabular}

real space, the correlations decay with distance since the Hamiltonian only couples neighboring lattice sites. An analogous statement does not apply in momentum space, where the interaction is highly non-local and can strongly couple momentum states that are far apart. A critical question there is how strong is this coupling relative to the single-particle splittings between the Bloch states. Nevertheless, the conclusion to draw from this work is that when studying 1D lattices using the DMRG algorithm, the real-space formalism remains the method of choice.

Xiang's results for the Hubbard model on a $4 \times 42$ D lattice are summarized in table 2. The results are shown for several values of $U / t$ and for several numbers of electrons. Where available they are also compared with results of Quantum Monte Carlo (QMC) and Stochastic Diagonalization (SQ) calculations. There are two key points to note. The first is that the method works very well for weak on-site repulsion. This should not be surprising, since it is exact when $U=0$. In that case, the exact solution - a Slater determinant of Bloch states up to the Fermi momentum - is a trivial one-state $\mathrm{kDMRG}$ wave function. The second point to note is that the method continues to work reasonably well even for stronger coupling, producing results that are competitive with those obtained using the two other popular many-body methods against which it was compared.

Several years later, Nishimoto and collaborators [46] reinvestigated the usefulness of the kDMRG method for treating the Hubbard model. They redid some of the calculations reported by Xiang and also carried out a much more systematic investigation, considering several different hopping scenarios. An important difference of their calculations relative to those of Xiang was in the blocking scheme. These authors applied White's blocking scheme, adding two sites at a time to enlarge the superblock. Xiang, in his kDMRG work, added only one site at a time. This makes it somewhat difficult to draw comparative conclusions, as the sizes of the superblocks that would arise in the two blocking approaches are different for the same number of states $m$ retained in each block.

We summarize here some of the key conclusions of ref. [46.

- In 1D with nearest-neighbor hopping, Xiang's results do not match theirs. Several 
possible explanations were put forth, including that Xiang's calculations may not have converged and that it could be a consequence of the different blocking schemes used in the two calculations.

- In $2 \mathrm{D}$, both the rDMRG and the kDMRG become less accurate with increasing dimensionality, albeit for different reasons. In real space, it relates to the level of off-diagonal coupling. In momentum space, it relates to the increased degeneracies and to the long-range nature of the interaction.

- The $\mathrm{kDMRG}$ is less dependent on the form of the hopping term. In real space, the method becomes rapidly worse as the hopping range increases; this is not the case in momentum space.

- In agreement with Xiang, they find that the accuracy of the DMRG method becomes worse with increasing strength $U$, scaled by the band width.

- In the case of the $4 \times 42 \mathrm{D}$ lattice at half filling, the $\mathrm{kDMRG}$ was found to be more accurate than the rDMRG for $U<8 t$.

\section{DMRG for finite Fermi systems}

The real-space DMRG has proven extremely successful in describing the physics of onedimensional systems with short-range interactions. As discussed in Sect. II.B.4, the method can be extended to 2D systems by selecting a particular path covering the lattice at the expense of introducing long-range interactions. These long-range interactions arise between neighboring sites in the 2D lattice, which may be located far away within the selected 1D path. The interplay between short-range and long-range interactions works to the detriment of the optimal accuracy of the 1D DMRG and to an increase in the memory requirements to implement the algorithm, because of the necessity of storing the matrix elements of long-range correlators. In practice, real-space 2D DMRG calculations were therefore restricted to small clusters or to ladders up to six legs [47.

The $\mathrm{kDMRG}$ represented a possible way to extend the DMRG methodology to larger dimensions. Unfortunately, the results obtained for the Hubbard models of 2D lattices were not very encouraging and the procedure was not pursued very much. However, the formal procedure developed by Xiang to reduce the number of matrix elements needed to store at each iteration was a critical step in the subsequent application of the DMRG methodology to finite Fermi systems, to which we now turn.

In finite Fermi systems one typically starts with a one- plus two-body Hamiltonian expressed in some single-particle basis,

$$
H=\sum_{i j} T_{i j} c_{i}^{\dagger} c_{j}+\sum_{i j k l} V_{i j k l} c_{i}^{\dagger} c_{j}^{\dagger} c_{l} c_{k}
$$

where $T_{i j}$ takes into account the kinetic energy and the mean-field interaction between particles and $V_{i j k l}$ represents the residual interaction.

A typical basis used in studies in quantum chemistry, nuclear physics, matter waves, etc. involves the use of the Hartree-Fock (HF) mean-field approximation. Other 
frequently-considered possibilities include the Kohn-Sham basis or approximate mean fields. As an example of the latter, nuclear structure calculations often use for simplicity a 3D harmonic oscillator basis or a basis comprised of the eigenstates of a Woods-Saxon potential, as approximations to the nuclear mean-field potential.

The first attempt to apply the DMRG to a 3D finite Fermi system was performed by White and Martin[20]. They proposed the use of the DMRG for ab initio calculations of the electronic structure of molecules. The method was further studied and refined for calculations in quantum chemistry and for application to quantum Hall systems 29, 30, 31, 32, 33. Other forms of the DMRG procedure were developed and applied to ultrasmall superconducting grains [24, 25] and the nuclear shell model[26, 27, 28. In the following subsections, we describe the progress achieved in each of these domains, as well as the difficulties and limitations of the procedures that were used.

\subsection{Quantum Chemistry}

$A b$ initio calculations in Quantum Chemistry (QC) are based on the approximate treatment of a system of $N$ electrons moving in a field created by $M$ fixed atomic nuclei and interacting through the Coulomb force. The electronic Hamiltonian of the system is

$$
H=\sum_{i=1}^{N}-\frac{\nabla_{i}^{2}}{2}-\sum_{i=1, \alpha=1}^{N, M} \frac{Z_{\alpha} e^{2}}{\left|r_{i}-r_{\alpha}\right|}+\frac{1}{2} \sum_{i \neq j=1}^{N} \frac{e^{2}}{\left|r_{i}-r_{j}\right|} .
$$

There are two possible avenues 38 for the treatment of the electronic Hamiltonian (23). One involves the use of density functional theory (DFT), which has been used extensively and with great success in the treatment of fairly large molecules. The other involves the use of Hartree-Fock (HF) mean-field theory as a starting point for an improved treatment of the electron-electron interaction.

In principle, the best means of treating the electron-electron interaction in a $\mathrm{HF}$ framework is through the full Configuration Interaction (CI) approach [38]. In this approach, the electron-electron interaction is exactly diagonalized in a basis of orthogonal states generated by the HF method. For problems that are not too large, this can be implemented through the use of diagonalization routines appropriate to sparse matrices, such as Lanczos or Davidson. With present computational resources, matrices of dimension $10^{7}$ to $10^{8}$ can be treated in this way, with little hope of a dramatic increase in the future. Other approximate but very accurate methods to treat the residual electronic interactions, such as Coupled Cluster Theory (CCT), are often used, but like the full CI they scale exponentially with the number of basis states, thereby imposing limitations on the Hamiltonian matrix dimensions that can be treated and permitting complete calculations for relatively small molecules only.

The DMRG method was proposed by White and Martin [20, 21] as an alternative procedure for approximately diagonalizing the residual electronic interaction, but in a 
much more computationally efficient way. Despite requiring a much slower polynomial computational effort, the method nevertheless has been shown to provide highly accurate results. More precisely, the DMRG computational effort scales as $O\left(n^{4} m^{2}\right)$ where $n$ is the total number of basis states and $m$ is the number of states retained in the iterative algorithm. This suggests that application of the DMRG algorithm to the field of QC could open up the possibility of a wide range of highly accurate molecular structure calculations, well beyond the limits of the full CI.

The usual way to generate a second quantized Hamiltonian in a finite molecular basis is to first consider a small number of atomic orbital states for each atomic nucleus, generating a local non-orthogonal basis, and then to use HF mean-field theory to produce an orthogonal molecular basis of $n$ orbitals, doubly-degenerate in the spin quantum number. The second-quantized Hamiltonian in this basis is

$$
H=\sum_{i j \sigma} T_{i j} c_{i \sigma}^{\dagger} c_{j \sigma}+\frac{1}{2} \sum_{i j k l \sigma \sigma^{\prime}} V_{i j k l} c_{i \sigma}^{\dagger} c_{j \sigma^{\prime}}^{\dagger} c_{l \sigma^{\prime}} c_{k \sigma},
$$

where $c_{i \sigma}^{\dagger}\left(c_{i \sigma}\right)$ is the creation (annihilation) operator of an electron in orbital $i$ with spin projection $\sigma$. The matrix $T$ is a radial integral of the electron kinetic energy and the electron-nucleus interaction in the HF basis, while the matrix $\mathrm{V}$ is a corresponding radial integral of the repulsive Coulomb interaction between electrons.

The key point in applying the DMRG procedure to QC problems is to treat each orbital as a "site" in a one-dimensional lattice. This site can be empty, singly occupied with spin up, singly occupied with spin down, or doubly occupied. The situation is analogous to the $\mathrm{kDMRG}$ and in fact it was the use of the Xiang trick for reducing the three-index operators to an effective one-index operator that made the procedure tractable in QC. The most complex operators that need to be calculated and stored in each iteration are those with two electronic creation and/or annihilation operators, requiring storage space of order $n^{2} m^{2}$.

As we will see below, a key issue, which is still not in our view fully resolved, is how to establish the optimal relative order among the orbitals. Two possibilities are to order the orbitals in terms of HF energies or to maximize the interactions between neighboring orbitals. A satisfactory resolution of this problem will ultimately lead to an optimized DMRG protocol for applications in QC.

As an illustration of the first tests of the method, we show in fig. 6 DMRG results for the methane molecule $\left(\mathrm{CH}_{4}\right)$ [21]. The system consists of 10 electrons moving in a minimal basis of 9 orbitals. The one- and two-body matrices in the Hamiltonian (24) were obtained following a HF calculation. The HF molecular orbits played the role of sites in a finite one-dimensional lattice and the finite DMRG algorithm was applied to the problem, with the ground state (GS) targeted. The DMRG results for the GS energy are plotted as a function of the number of states $m$ retained in the iterative procedure. For comparison, the figure also includes the $\mathrm{CAS}(\mu, \nu)$ results corresponding to diagonalization of the Hamiltonian (24) in a Complete Active Space of $\nu$ valence orbitals and $\mu$ active electrons. 


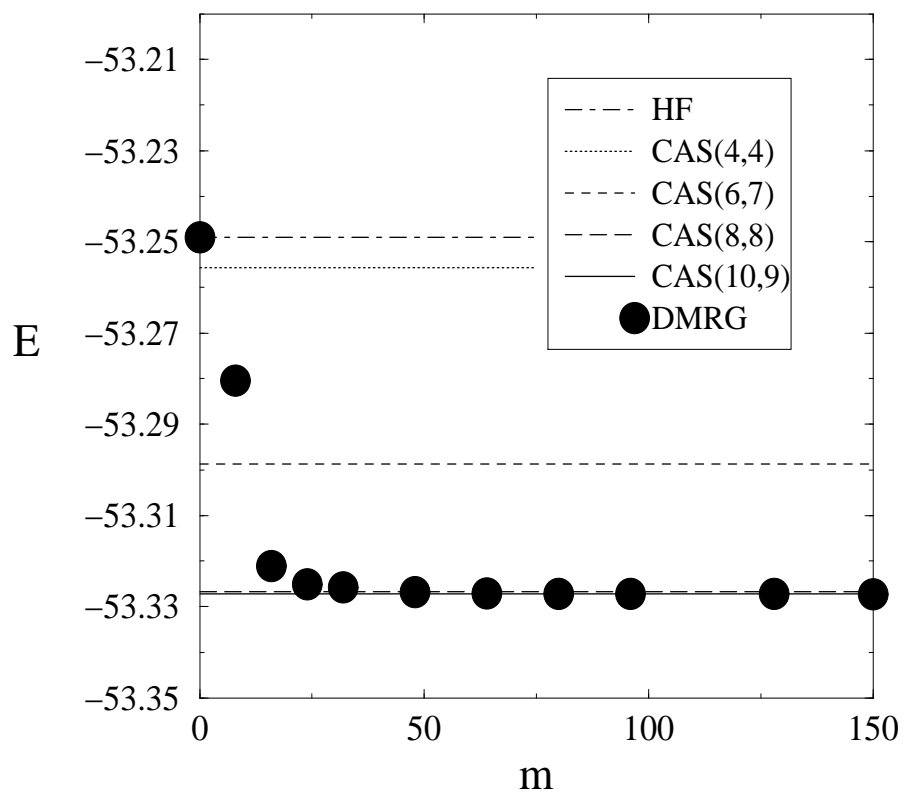

Figure 6. Ground-state energy $E$ of $\mathrm{CH}_{4}$ as a function of the number of states retained $m$ at each DMRG iteration. The lines are the Hartree-Fock and CAS results. Taken from [21]. Reprinted by permission of John Wiley \& Sons, Inc.

The DMRG results exponentially converge to the exact results of CAS $(10,9)$ in which the complete active space of 9 molecular orbits is taken into account. For rather small $m$ values $(\sim 50)$ the DMRG results are already comparable to CAS $(8,8)$ in which two electrons are frozen in the lowest HF orbital.

The influence of the choice of basis on the global procedure was also investigated in [21], with a focus on the singlet state of the H-He molecule. The standard definition of the molecular orbits based on HF mean field approximation was compared with the DFT definition of natural orbitals in two different approximations - the Local Density Approximation and the Generalized Gradient Approximation. The results obtained were fairly insensitive to the choice of basis, showing similar convergence properties.

The efficiency of the DMRG procedure in QC has been further studied in ref. 22]. Though the method was found to perform very well for the molecules $\mathrm{Be}_{2}$ and $\mathrm{HF}$, comparison with full CI results for the $\mathrm{N}_{2}$ molecule did not produce satisfactory results. The reasons for this failure were not conclusive, but there were indications that a non-optimal ordering of orbitals may have played an important role.

A very careful study of all of the ingredients that define the performance and efficiency of the DMRG algorithm in QC was reported in ref. [23, where the reader can also find a detailed and pedagogical presentation of the method. The subject of the orbital ordering was addressed in this work, with the idea of minimizing the range of the interaction matrix elements in order to render the correlation length of the system a minimum. After trying various prescriptions, the authors came to the conclusion that the simplest method was to minimize the band-with of the one-body matrix elements 
Table 3. DMRG results for the water molecule for various values of $m$, compared with three levels of approximation in CCT. Taken from [23]. Reprinted by permission of the American Institute of Physics.

\begin{tabular}{lrr}
\hline$m$ & $E / H$ & $\delta E / m H$ \\
\hline 100 & -76.2545 & 2.1 \\
200 & -76.2559 & 0.71 \\
300 & -76.25632 & 0.32 \\
400 & -76.256477 & 0.157 \\
500 & -76.256540 & 0.094 \\
600 & -76.256592 & 0.042 \\
750 & -76.256617 & 0.017 \\
900 & -76.256624 & 0.004 \\
\hline$C C T$ & -76.252503 & 4.131 \\
$C C T(T)$ & -76.255907 & 0.727 \\
$C C T(T Q)$ & -76.256846 & 0.202 \\
\hline
\end{tabular}

$T_{i j}$. They found that the symmetric reverse Cuthill-McKee reordering method could bring the $T$ matrix to a block diagonal form, avoiding long-range hopping terms.

The example of the water molecule, which had been treated by White and Martin in [20] as a first test of the DMRG method in QC, was re-investigated in [23], to compare the accuracy of the DMRG not only with a full CI calculation but also with current CCT approximations. This problem involves 8 electrons moving in 25 active orbits (the $1 \mathrm{~s}$ orbit was frozen). We reproduce in table 3 their results. The exact GS energy given by a complete CI diagonalization is $-76.256634 H$. CCT(T) and CCT(TQ) refer to Coupled Cluster calculations with triples and with triples and quadruples, respectively. It can be seen from the results that for $m=400$ the DMRG already has comparable accuracy to CCT(TQ). Furthermore, and most importantly, since DMRG is a variational theory we can be sure that the numerical result it yields is an upper bound to the GS energy. This is not the case for CCT, as seen from the CCT(TQ) result for which there is overbinding of $0.2 \mu \mathrm{H}$.

Analysis of larger molecules, such as $C_{2} H_{4}$, indicates that it is difficult for the DMRG method to capture long-range electron correlations because of the sequential procedure it uses for adding orbitals. Nevertheless, the fact that the DMRG results obtained in ref. 23] were comparable to the CCT results including quadruples suggests that it should be taken seriously in such calculations, with possible improvements now to be discussed.

A difficulty in drawing firm conclusions from the studies just discussed is that exact results from full $\mathrm{CI}$ calculations were not available in all cases, making it impossible to confidently assess the true accuracy of the DMRG and CCT calculations. One of the control parameters of the DMRG method is the number of states $m$ retained in the algorithm. The variational properties of the DMRG assure that the GS energy decreases, or at worst stays constant, with increasing $m$ values. Indeed, in many 

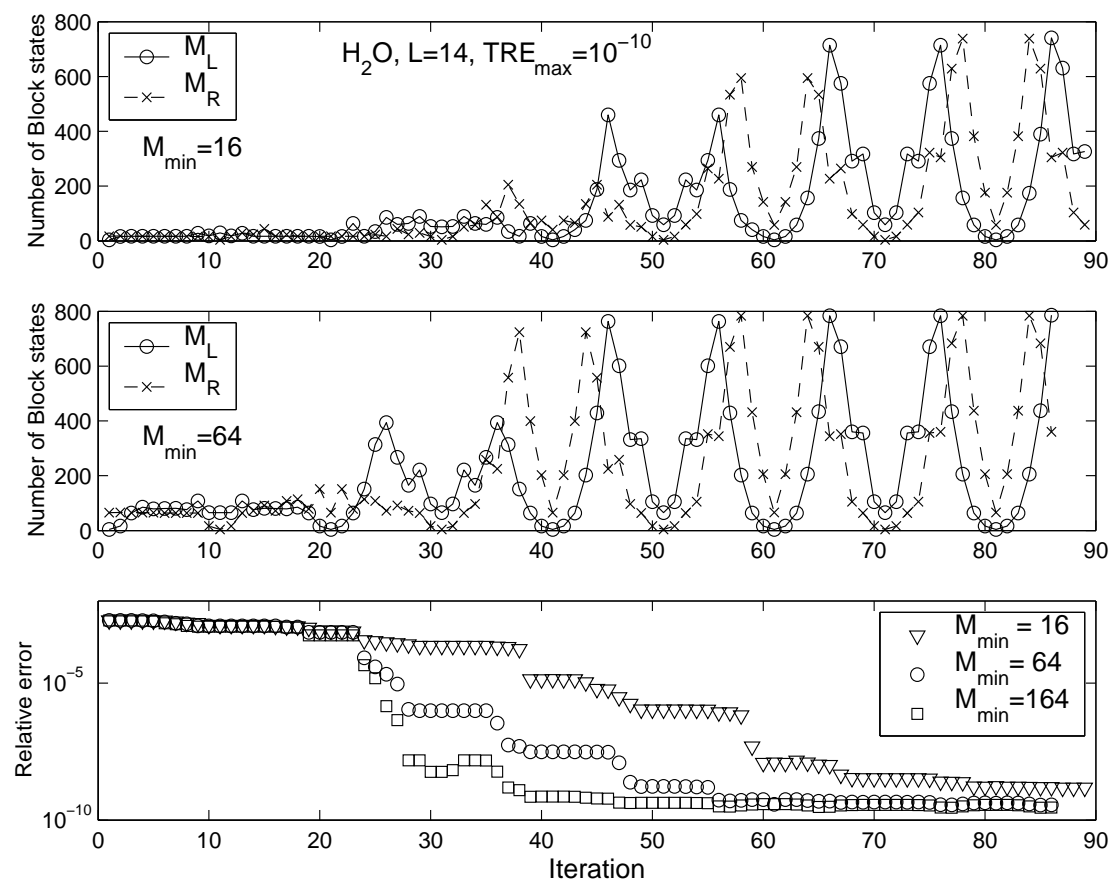

Figure 7. Dynamically selected number of left and right blocks states for two values of the minimum threshold $m_{\min }=16,64$ and the relative error as a function of iteration for three values of $m_{\min }=16,64,164$. In all cases the 10 electrons of the $\mathrm{H}_{2} \mathrm{O}$ molecule were correlated in the double-zeta water model with 14 orbitals, and $T R E_{\max }=10^{-10}$ was set in advance of the calculations. Taken from ref. [51]. Reprinted by permission of the American Physical Society.

cases an exponential or modified exponential [23] convergence with $m$ has been found. Nevertheless, even in such cases it is difficult to correlate the number of states $m$ with the final precision of the procedure. In ref. [51], a new method for determining the accuracy of the DMRG in advance, the Dynamical Block State Selection (DBSS) method, was proposed. The truncation error $(T R E)$ in each iteration is $T R E=1-\sum_{\alpha} \omega_{\alpha}$, where $\omega_{\alpha}$ are the eigenvalues of the reduced density of the block in descending order. Keeping the same number $m$ of states in each iteration induces fluctuations in the TRE, making it impossible to infer the final accuracy of the procedure. The DBSS method inverts the procedure for selecting states by defining a maximum truncation error $T R E_{\text {max }}$ at the start, which amounts to a dynamical selection of the number of states kept in each iteration. The DBSS is supplemented by the selection of a lower threshold $m_{\min }$ for the number of states retained. The first and second panels of figure [7 extracted from ref. [51], show the number of states dynamically selected in each iteration for two values of $m_{\min }$ in a calculation for a water molecule with 10 electrons and 14 orbitals. The third panel shows the relative error in the GS energy, defined as $\left(E_{D M R G}-E_{C I}\right) / E_{C I}$.

It is interesting to note that even though the maximum number of states does not depend on $m_{\min }$ the rate of convergence is faster for larger $m_{\min }$ values. In the example of the water molecule, the HF single particle levels were ordered with increasing values 
of energy. In other test cases, e.g. for the $\mathrm{CH}_{2}$ molecule [51, this ordering led to a local minimum and only by changing the ordering was it possible to obtain global convergence.

The problem of the single-particle ordering was investigated recently in ref. [52], where a protocol for the initialization of the procedure and for an optimal ordering of the levels was proposed. Using concepts borrowed from Quantum Information Theory, it was shown that the optimal ordering corresponds to locating the levels with maximum entropy at the center of the chain. This ordering is not always equivalent to the CuthillMcKee ordering that had been proposed in [23].

There have been several other applications of the DMRG algorithm in Quantum Chemistry to large polymers [53, 54, 55, 56, 57, 58, 59, 60, 61]. These applications have typically utilized the original real-space DMRG algorithm building on the fact that polymers can be represented either by a chain or by another cyclic structure. Since our focus is on applications that require the machinery appropriate to finite Fermi systems, we will not discuss these applications here.

\subsection{Ultrasmall superconducting grains}

In 1959, Anderson 62 posed the question of what would be the lower size limit for a metallic grain to sustain superconductivity. Since the average level spacing $d$ in a metallic particle is inversely proportional to its volume, for small enough particles (namely when $d$ is of the order of the bulk gap $\Delta$ ) the scattering of pairs across the Fermi surface ceases to be an efficient mechanism for lowering the energy of the system. At this point superconductivity should therefore disappear. Anderson estimated the critical grain size to be in the range of nanometers. This old question has recently been revived in the context of experiments performed by Ralph, Black and Tinkham 63. on ultrasmall Aluminum grains. They succeeded in constructing a Single Electron Transistor whose central island was an Al grain of a few nanometers with an estimated mean level spacing $d \sim 0.4 \mathrm{mev}$, of the order of the bulk gap. The experiments showed the existence of a spectroscopic gap that could be driven to zero by the application of magnetic fields.

The Hamiltonian proposed in 64] to study the physics of such small grains is of the reduced BCS form,

$$
H=\sum_{j=1}^{\Omega} \sum_{\sigma= \pm}\left(\epsilon_{j}-\mu\right) c_{j \sigma}^{\dagger} c_{j \sigma}-\lambda d \sum_{i, j=1}^{\Omega} c_{i+}^{\dagger} c_{i-}^{\dagger} c_{j-} c_{j+},
$$

where $i, j=1,2, \ldots, \Omega$ label the single-particle energy levels, which are assumed to be equally spaced $\left(\epsilon_{j}=j d\right)$, and $c_{j \sigma}$ are the electron annihilation operators associated with the two time-reversed states $|j, \sigma=+\rangle$ and $|j, \sigma=-\rangle$. Finally, $\mu$ is the chemical potential and $\lambda$ is an adimensional BCS coupling constant, whose appropriate value for $\mathrm{Al}$ grains is 0.224 65. The systems described by this Hamiltonian have been treated at half filling for which the number of pairs $N$ equals the number of levels $\Omega$. 
It soon became clear that the standard BCS approximation, which so accurately describes superconductivity for sufficiently large systems, is not well suited to treat the systems of physical interest, for which $N \sim 100$. In such systems, there are strong number fluctuations proportional to $\sqrt{N}$. Several levels of approximations were thus applied to the Hamiltonian (25) , to see whether any could describe the physics of pairing in finite systems. In increasing order of complexity, they included i) BCS approximation with parity projection [64], ii) Lanczos diagonalization up to $\Omega=23$ with finite-size corrections [66], and iii) number-projected BCS [67] approximation. All of these approximations predicted a phase transition from superconducting behavior to a normal state at a critical grain size, and in all cases the transition was abrupt. In contrast, the DMRG method - when applied in a somewhat modified form [24] showed that the crossover as a function of decreasing grain size had a smooth reduction in pairing correlations.

Following completion of [24, the authors learned that the Hamiltonian (25) had been solved exactly by Richardson during the 1960s 68. This made it possible to carry out a comparison of the DMRG results with those obtained from Richardson's exact solution. For the largest system treated, with 400 doubly-degenerate levels, the condensation energy was $E_{D M R G}^{C}=-22.5168$ with a predicted relative error of $10^{-4}$. Richardson's exact result was $E_{\text {exact }}^{C}=-22.5183141$, confirming the accuracy of the phDMRG. and showing that the relative error predicted by the DMRG method was in fact appropriate. On this basis, it is clear that the DMRG method provides an essentially exact description of the physics of pairing in finite systems and that the correct crossover from superconducting to the normal state is indeed smooth and not abrupt.

The modifications implemented in the DMRG method for its application to small metallic grains were based on the fact that in a finite Fermi system the Fermi energy $\left(E_{F}\right)$ naturally separates the single-particle space into a primarily occupied subspace and another primarily empty subspace. The method that emerges from such a construction was called the particle-hole DMRG (phDMRG), which we now briefly describe.

Consider the set of single-particle levels shown in fig. 8. Note that they are divided into two sets. The particle levels are those above the Fermi surface, which should be essentially empty. The hole levels are those below the Fermi surface and they should be essentially filled. In the phDMRG method, one first defines particle and hole blocks from the levels nearest to the Fermi surface, and then gradually grows those blocks by adding levels progressively further away. The figure is meant to signify that we have already created particle and hole blocks involving the first two available levels, respectively, and are enlarging them by adding the third level(s). The particle block then serves as the medium for the hole block and the hole block as the medium for the particle block as we implement the DMRG truncation strategy. This process is continued until all particle and hole levels have been treated.

The phDMRG procedure, as just described, is related to the infinite DMRG algorithm in that sweeping is not implemented. It is extremely difficult to implement 


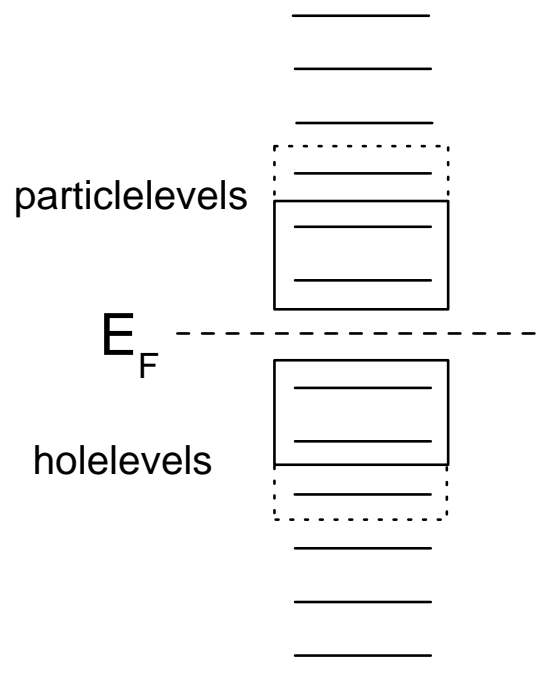

Figure 8. Schematic illustration of the phDMRG method for finite Fermi systems. The single-particle levels are divided into primarily occupied hole levels and primarily empty particle levels. The first two levels above the Fermi energy $E_{F}$ have been formed into a particle block and the first two below have been formed into a hole block. The third levels above and below are being added to form enlarged blocks.

sweeping with particle and hole blocks while at the same time preserving the four block structure of the method. As such, it is expected that the phDMRG will only work well for systems where the correlations fall off rapidly as we move away from the Fermi surface. The key point is that at the beginning of the iterative procedure the levels that are outside the particle-hole superblock do not impact on the selection of the optimal state(s) with which to truncate the two blocks. This is a recurring limitation of the infinite algorithm, which can be partially overcome by combining the DMRG truncation strategy with the use of effective interaction theory. In the application to ultrasmall superconducting grains, this was implemented through a phenomenological renormalization of the pairing strength at each iteration. When the system described by the pairing Hamiltonian (25) for $\Omega$ levels at half filling is considered in BCS approximation, the bulk gap is given by $\Delta_{\Omega}=d \Omega / 2 \sinh (1 / \lambda)$. The effect of the levels outside a superblock that consists of $2 n$ levels can thus be taken into account in an average way by introducing an effective coupling strength $\lambda_{n}$ - corresponding to step $n$ of the iteration - such that the gap in the superblock being considered equals the bulk gap. This is guaranteed if the renormalized coupling strength $\lambda_{n}$ is chosen according to

$$
\sinh \lambda_{n}=\frac{2(n+1)}{\Omega} \sinh \lambda .
$$

Note that the pairing strength assumes its full value when all levels are included, i.e., when $n=\frac{\Omega-1}{2}$.

The effect of renormalizing the coupling strength in this way can be seen in table 4. where we show results for the condensation energy in units of the mean level spacing, 
Table 4. The effect of renormalization on the results of phDMRG calculations for ultrasmall superconducting grains.

\begin{tabular}{|c|c|c|c|}
\hline$m$ & $E_{\text {bare }}^{C} / d$ & $E_{\text {dressed }}^{C} / d$ & $\operatorname{dim} \mathcal{H}_{100, m}$ \\
\hline 40 & -40.32502 & -40.49884 & 1246 \\
50 & -40.44623 & -40.50014 & 2108 \\
60 & -40.46887 & -40.50061 & 3032 \\
70 & -40.48878 & -40.50068 & 3622 \\
80 & -40.49588 & -40.50072 & 4820 \\
90 & -40.49815 & -40.50074 & 6306 \\
100 & -40.49919 & -40.50075 & 7778 \\
110 & -40.49983 & -40.50075 & 9720 \\
\hline
\end{tabular}

without renormalization $E_{\text {bare }}^{C}$ and with renormalization $E_{\text {dressed }}^{C}$, for different values of the number of states $m$ maintained. The system considered has $\Omega=100$ at half filling and the bare coupling constant is $\lambda=0.4$, for which the system is well into the superconducting phase. In the last column, we show the dimension of the largest superblock required in the iterative process, which should be compared with the total dimension of the problem, $10^{29}$.

As can be seen from the table, there is a significant improvement in convergence when renormalization of the coupling constant is implemented in the phDMRG algorithm. For $m=100$, for which the largest matrix diagonalized had a dimension of roughly 8,000 , the condensation energy (column 3 ) has converged to seven significant figures, while the standard phDMRG procedure (column 2) without renormalization is still far from convergence. The results of the complete calculation for ultrasmall superconducting grains are shown in fig. 9. The condensation energies for even and odd systems are displayed as a function of the mean level spacing in units of the bulk gap. The solid lines correspond to the numerically exact phDMRG calculation and the squares and triangles, for even and odd systems, respectively, correspond to the PBCS calculations of [65]. The phDMRG results were obtained with $m=60$ with an estimated relative error for the largest system considered ( $\Omega=400$ levels) of $10^{-4}$. The phDMRG results are consistently below the PBCS results, representing a considerable gain in correlation energy. Most importantly, while the PBCS results suggest an abrupt change of the condensation energy at a critical value of the level spacing, the phDMRG results unveiled the true nature of the transition. There is a very smooth crossover as the size of the grains decrease, from superconducting grains to metallic grains with strong pairing fluctuations.

The fact that BCS approximation ceases to provide a good approximation for small enough grains suggests that other phenomena associated with superconductivity may also be strongly modified in that regime. One example is the Josephson effect, whereby correlated Cooper pairs tunnel from one superconducting system to another due to the differences in phases of their respective order parameters. When BCS approximation breaks down, it is no longer meaningful to speak of superconducting phases, raising the 
The Density $M$

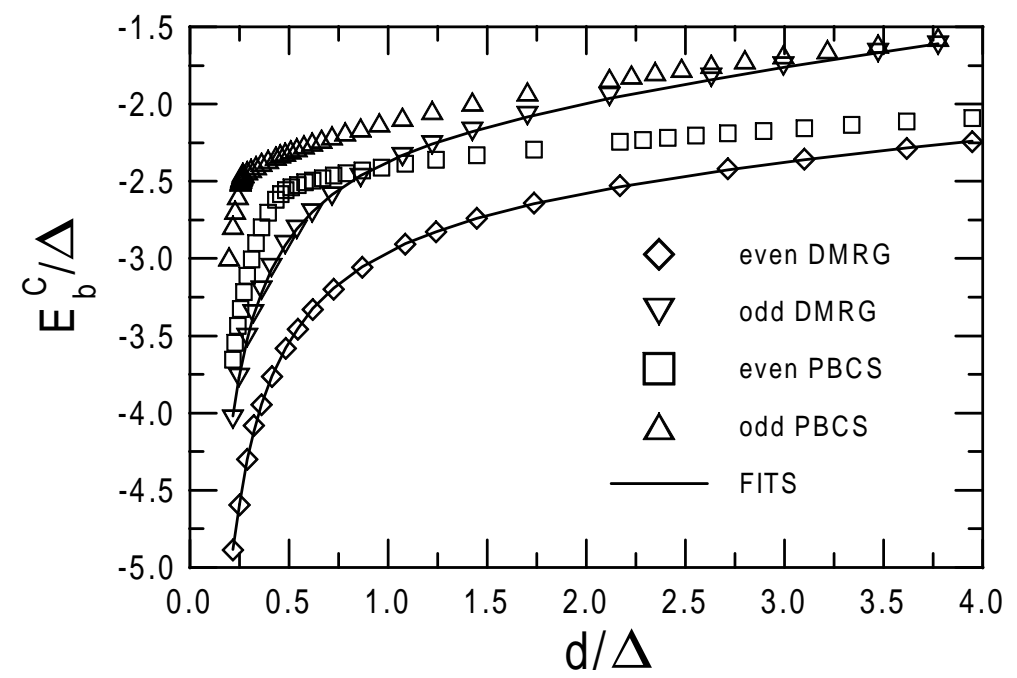

Figure 9. Ground state condensation energies $E_{b}^{C}(b=0,1)$ as a function of $d / \Delta$ for $\lambda=0.224$.. $\Omega$ ranges from 22 ( 23) up to 400 (401) for even (odd) grains and $m=60$. The PBCS results are those of ref. 67. Taken from 24. Reprinted by permission of the American Physical Society.

question of what is the fate of the Josephson effect between two small superconducting grains. This question has recently been addressed in ref. 69].

To study the physics of two very small grains, it is necessary to have a computational framework that can accurately describe the dynamics. From what we discussed earlier, BCS approximation is inadequate in a regime in which the two grains are sufficiently small. The Richardson approach, which gives the exact solution for individual grains, no longer applies when there are two distinct grains with tunnelling between them. The phDMRG approach, which had been used successfully for individual grains, can be naturally generalized to a two-grain scenario and might therefore provide a useful framework for an accurate treatment of the tunnelling phenomenon. In ref. [69, the two-grain phDMRG method was developed and applied to this problem. The basic idea of the method is to add a particle level and a hole level for one type of grain and then to follow that by adding a particle level and a hole level of the other type, gradually moving away from the Fermi surface. At each step, truncation is implemented in the particle block (which includes levels from both grains) and the hole block (likewise with levels from both grains) based on the associated reduced density matrices. This process is continued until all levels have been treated.

There are two variables that define the two-grain problem. One is the size of the grains, or equivalently the number of single-particle levels that contribute. The second is the Josephson coupling, namely the strength of the term in the Hamiltonian that governs pair tunnelling.

For the purposes of this review, perhaps the key point that emerged from the study concerns the range of validity of the two-grain DMRG method as a function of these two variables. This is schematically illustrated in fig. 10. together with an analogous 


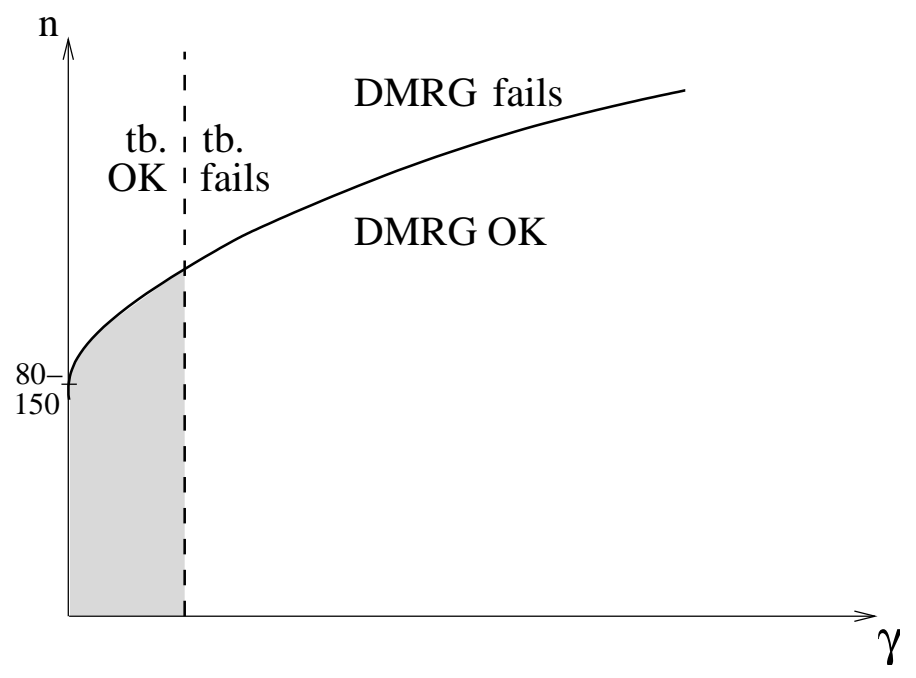

Figure 10. Schematic illustration of the regimes of validity of the two-grain DMRG method and the tight-binding (tb) method in the description of tunnelling between two superconducting grains. $\gamma$ represents the inter-grain coupling and $n$ the number of energy levels. Taken from Ref. 69.

illustration of the range of validity of the tight-binding (tb) method.

The tight-binding method - often called the weak-coupling method - assumes that the coupled system can be represented as a product of the ground states of two decoupled systems, which themselves can be obtained from the ordinary one-grain DMRG method. As the figure indicates, this should only work well for very weakly-coupled grains.

The two-grain DMRG method has a very different range of validity. It does not in general work well for weakly-coupled grains, except if the grain sizes are sufficiently small. This can be understood from the following argument. The DMRG method, in all of its manifestations, depends critically on the presence of correlations between the block and the medium in order to effectively truncate the space. When the two grains are very weakly coupled, there is no natural way to truncate the block, as all states are of comparable importance. Of course, if the number of levels is sufficiently small, the number of states $m$ required to adequately describe it is also very small and thus the DMRG can still well approximate it. These points are all nicely borne out in the calculations reported in ref. [69.

\section{2D Electrons in a High Magnetic Field}

Electrons in high magnetic fields are constrained to move in an effective two-dimensional space characterized by the Landau orbits. Depending on the filling of the orbits, various phases have been predicted and subsequently observed. The Fractional Quantum Hall (FQH) effect, discovered for various fractional fillings in the lowest Landau level, was followed by the observation of different exotic phases, like stripes, bubbles, Wigner crystallization, etc... 
An important understanding of these phases has been obtained by exact diagonalization for small systems [70] and by Quantum Monte Carlo studies [71]. Clearly, there are strong limitations to both methods. Large scale diagonalization can only handle systems with $N_{e} \sim 10$ electrons, while Monte Carlo calculations only provide the bulk properties of the ground state, with the accuracy of its results being limited by the well-known sign problem.

The DMRG offers here a great opportunity to go well beyond these limits in the study of 2D electrons in the lower Landau levels for different filling factors. As in other applications of the DMRG to $2 \mathrm{D}$ systems, the main problem is the mapping onto an effective 1D path. In ref. 29], the authors proposed the use of the eigenstates of free electrons in a perpendicular magnetic field in the Landau gauge to represent the local orbitals that play the role of the single-electron states. The Hamiltonian representing the Coulomb repulsion among the electrons in the local orbital basis is written as

$$
H=S \sum_{n} c_{n}^{\dagger} c_{n}+\frac{1}{2} \sum_{n_{1} n_{2} n_{3} n_{4}} A_{n_{1} n_{2} n_{3} n_{3}} c_{n_{1}}^{\dagger} c_{n_{2}}^{\dagger} c_{n_{4}} c_{n_{3}},
$$

where $S$ is a classical Coulomb energy and $c_{n}^{\dagger}$ creates an electron in the single-particle state $n=1, \ldots, M, M$ being the total number of single-particle states in the unit cell, and $A$ is the interaction matrix of the Coulomb force (for details see 32]).

The Hamiltonian (27) represents a strongly interacting electron system with degenerate single-particles states. While the Landau basis provides a natural mapping to a $1 \mathrm{D}$ system by choosing the single-particle states $n$ as the effective sites in a $1 \mathrm{D}$ lattice, the complete degeneracy of the states without the existence of a Fermi energy impedes the separation into particle and hole blocks and the identification of a region of the single-particle space with strong correlations. Furthermore, the interaction matrix $A$ is "long range" in the single-particle space, in the sense that it has finite matrix elements between distant sites in the 1D lattice.

The finite algorithm DMRG was applied to the system described by the Coulomb Hamiltonian (27) in the basis of Landau orbits $N=0,1$ and 2, to study the ground state properties and to determine the phase diagram as a function of the filling factor $\nu=N_{e} / M[31$, 33].

In the warm up, the first left block was constructed from the states $n=1,2$ and the first right block from the states $n=M, M-1$. The sites with $n=3$ and $n=M-2$ were subsequently added to the left and right blocks, respectively, in the first iteration, with a truncation based on the eigenvalues of the density matrix implemented to retain $m$ states. The method continued in this way until all sites were taken into account, completing the warm up. Then, several sweeps were performed until convergence in the ground-state energy was obtained. A typical example of the exponential decrease of the density matrix eigenvalues is given in fig. 11] for a system of 18 electrons in $M=54$ single-particle states. Retaining $m=200$ states in each iteration leads to a global accuracy of $10^{-4}$. 


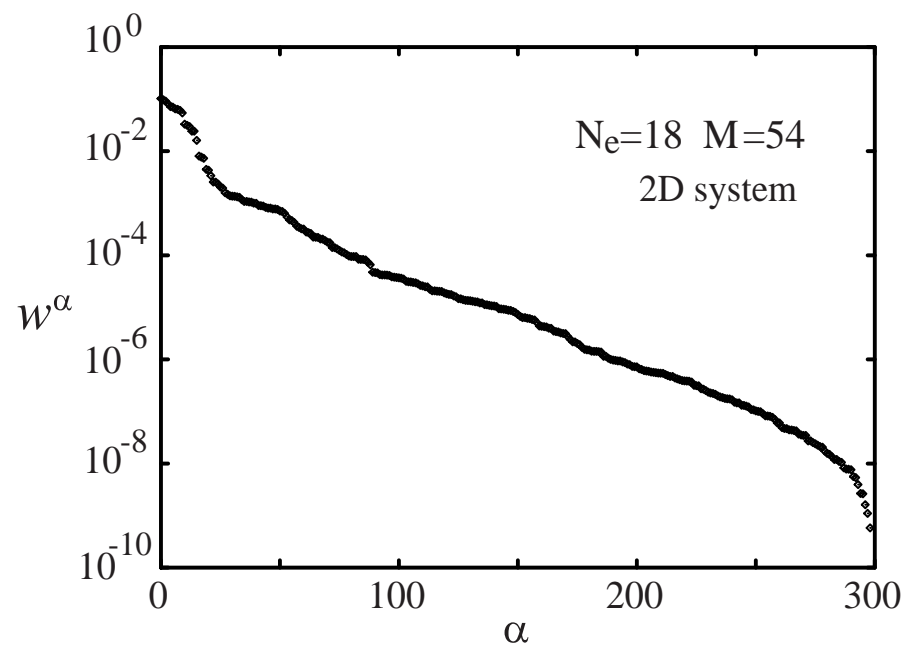

Figure 11. Eigenvalues $w^{\alpha}$ of the density matrix for a system with 18 electrons on 54 local orbitals. Taken from [32. Reprinted by permission of IOP Publishing Ltd.

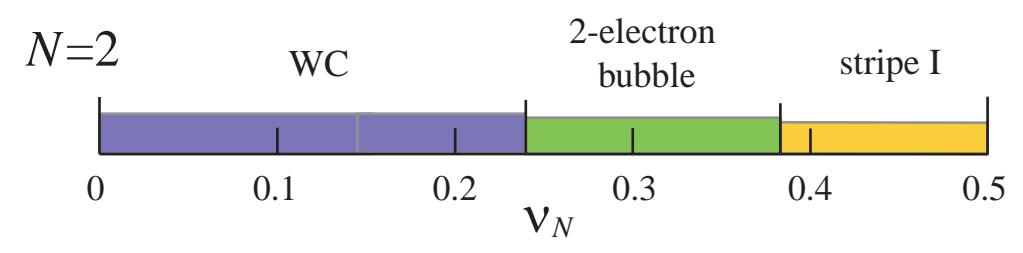

Figure 12. The ground-state phase diagram of the third-lowest Landau level obtained from a DMRG calculation with up to 25 electrons. Taken from 33

To identify the properties of the ground state for the different filling factors, the expectation value of the pair correlation function in the DMRG wave function,

$$
g(\mathbf{r}) \equiv \frac{L_{x} L_{y}}{N_{e}\left(N_{e}-1\right)}\left\langle\Psi\left|\sum_{i \neq j} \delta\left(\mathbf{r}+\mathbf{R}_{i}-\mathbf{R}_{j}\right)\right| \Psi\right\rangle,
$$

was used. Here, $\mathbf{R}_{i}$ is the guiding center of the $i$ th electron, $\mathbf{r}$ is the relative distance between the pair of electrons and $L_{x, y}$ are the sides of the unit cell.

A thorough study of the phase diagram of $2 \mathrm{D}$ electrons in the lowest three Landau levels was carried out in [31, 33. We present in fig. 12, as an example, the phase diagram of the third Landau level $(N=2)$ 33. The diagram shows a Wigner Crystal for low filling factors up to $\nu=0.24$, after which a transition to a 2-electron bubble phase takes place. There is a second transition to a stripe phase at $\nu=0.38$. The properties of the ground state are nicely displayed in fig. [13, where we can see the quite marked geometrical characteristics of the three phases in the pair correlation function.

The finite algorithm DMRG in the local Landau basis has been shown to constitute a powerful tool for investigating the physics of $2 \mathrm{D}$ electrons in the lowest Landau levels. Further optimization of the method could allow the study of larger systems and the investigation of the behavior of the systems close to the phase transitions. 
(a)

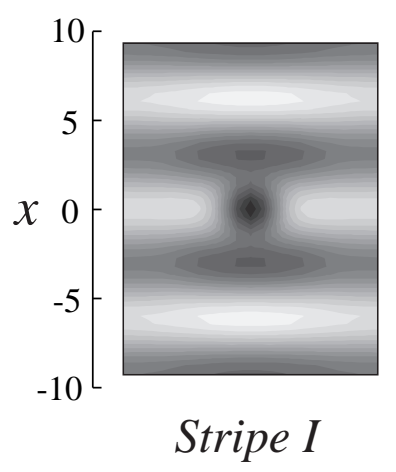

(b)

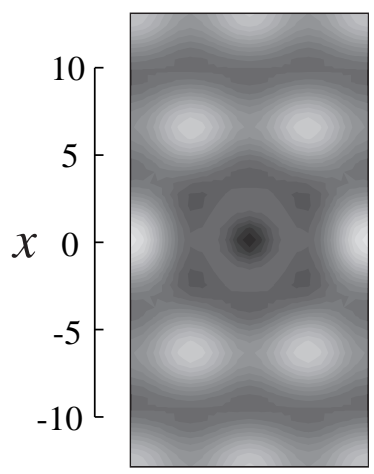

Bubble (c)

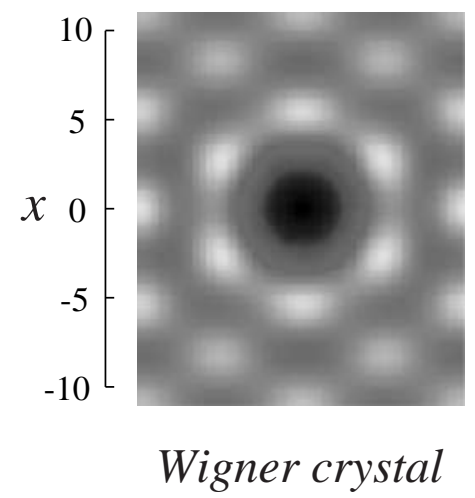

Figure 13. Pair correlation function of (a) a stripe state, (b) a two-electron bubble state, and (c) a Wigner crystal. Taken from 33.

\subsection{The DMRG in nuclear structure}

The successes of the DMRG method in Quantum Chemistry, in the treatment of ultrasmall superconducting grains and in the study of $2 \mathrm{D}$ electronic systems suggests that it might also prove useful in the treatment of another finite Fermi system, the atomic nucleus. The typical shell-model problem in nuclear structure involves solving the Schrödinger equation with a one- and two-body interaction in a spherical shellmodel basis. The system usually involves two kinds of interacting particles, neutrons and protons, with a Hamiltonian

$$
H=H_{p}+H_{n}+V_{p n}
$$

that includes three terms - one that acts solely in the proton sector, one that acts solely in the neutron sector, and the proton-neutron interaction.

For heavy enough nuclei and for nuclei with a fairly large number of active (or valence) nucleons, exact diagonalization of the shell-model Hamiltonian is not feasible. As an example, to treat the $J^{\pi}=2^{+}$states in the deformed nucleus ${ }^{154} \mathrm{Sm}$ assuming an inert ${ }^{132} S n$ core, 12 valence neutrons in the orbits $3 f_{7 / 2}, 3 p_{3 / 2}, 1 h_{9 / 2}, 1 i_{13 / 2}, 3 p_{1 / 2}, 2 f_{5 / 2}$ and 8 valence neutrons in the orbits $1 g_{7 / 2}, 2 d_{5 / 2}, 1 h_{11 / 2}, 2 d_{3 / 2}, 3 s_{1 / 2}$ requires diagonalization in a basis involving $3.46 \times 10^{14}$ states, well beyond the limits of the best diagonalization routines. Might it be possible, however, to iteratively obtain accurate approximate solutions to the shell-model problem using the DMRG strategy? This question has been discussed in several recent works based on the particle-hole algorithm, as we now briefly review.

A characteristic feature of nuclear structure is the presence of several competing collective structures, most importantly those associated with the pairing and quadrupole fields. In refs. [26, 27], the phDMRG was applied to a test model involving both pairing 


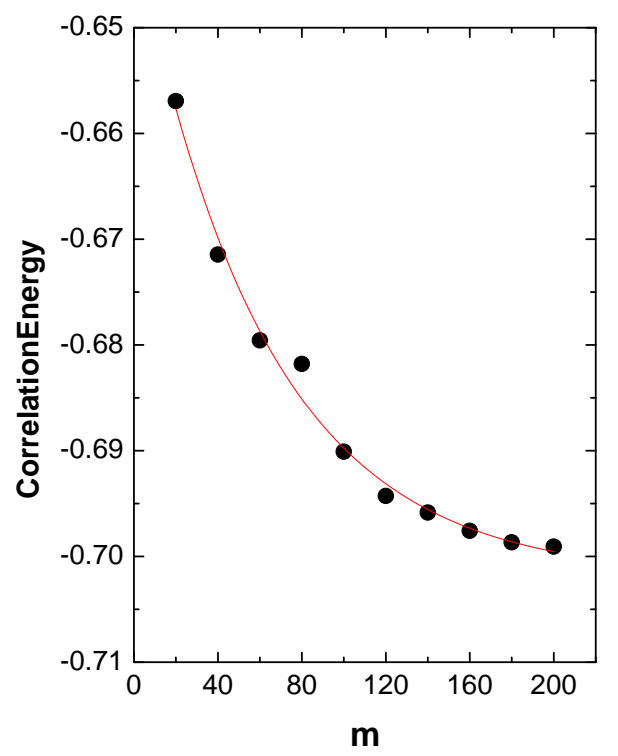

Figure 14. DMRG correlation energies as a function of $m$ for a system of 10 particles in a $j=25 / 2$ orbit subject to a Hamiltonian with $\chi=1, g=0.1$, and $\epsilon=0.1$. The solid line represents an exponential fit to the DMRG results. Taken from [27. Reprinted by permission of the American Physical Society.

and quadrupole collectivity to assess whether the algorithm is able to handle their interplay. For simplicity, the calculations were carried out in a model involving one type of nucleon in a large single-j shell, interacting via a $P+Q$ Hamiltonian

$$
H=-\chi Q \cdot Q-g P^{\dagger} P-\epsilon \sum_{m}|m| a_{j m}^{\dagger} a_{j m},
$$

where

$$
Q_{\mu}=\sum_{m}\left\langle j m+\mu\left|Q_{\mu}\right| j m\right\rangle c_{m+\mu}^{\dagger} c_{m}
$$

and

$$
P^{\dagger}=\sum_{m}(-)^{j-m} c_{m}^{\dagger} c_{-m}^{\dagger}
$$

The third term in the Hamiltonian splits the levels of the single-j shell into a set of equally-spaced levels of oblate character, with the largest $|m|$ value lowest. Because of that term, the Hamiltonian is not in general rotationally invariant and thus its eigenstates do not conserve angular momentum. This unphysical assumption was introduced so that each single-particle orbit would be doubly degenerate, greatly facilitating the use of an iterative computational algorithm in the test calculation.

The first results reported were for a case of 10 particles in a $j=25 / 2$ orbit. The calculations were restricted to states with total angular momentum projection $M=0$, for which the model admits 109,583 states, well within the limits of standard diagonalization routines. Thus, for this problem comparison of the DMRG results with the exact results was feasible. The results we show were obtained for the case 


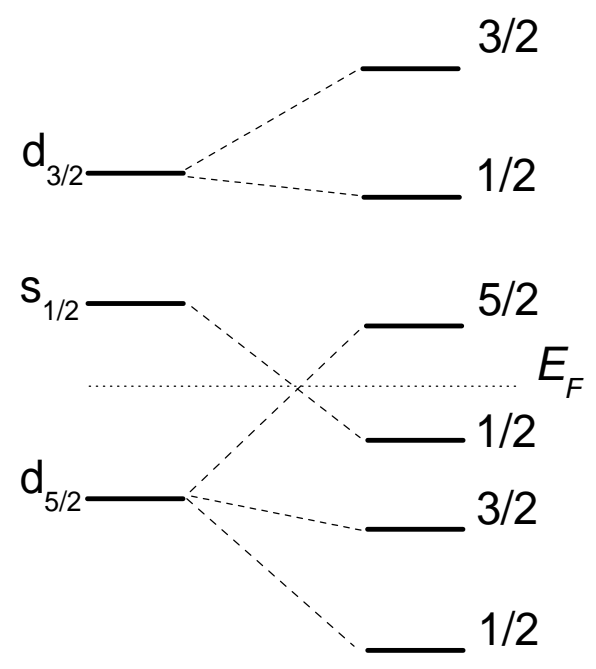

Figure 15. Schematic illustration of the splitting of the spherical single-particle levels of the $2 s-1 d$ shell into a set of doubly-degenerate levels by an axially-deformed Hartree-Fock calculation. The dashed line represents the Fermi energy $\left(E_{F}\right)$, which separates the particle levels from the hole levels. Each doubly-degenerate level is labelled by its angular momentum projection on the intrinsic z-axis. Taken from ref. 28.

of $\chi=1, g=0.1, \epsilon=0.1$, for which Hartree Fock Bogolyubov (HFB) approximation shows a well-defined superconducting solution. As such, the test Hamiltonian describes a scenario in which pairing and quadrupole correlations indeed compete.

Figure 14 compares the correlation energies, defined as the gain in ground-state energy relative to Hartree Fock approximation, from the DMRG method and exact Lanczos diagonalization. For comparison, the exact and HFB values are $E_{\text {corr }}^{\text {Exact }}=$ -0.70633 and $E_{\text {corr }}^{H F B}=-0.20641$. By an $m$ value of 200, the DMRG method produced more than $99 \%$ of the exact correlation energy, a dramatic improvement over HFB approximation which only achieved $28 \%$. The largest superblock matrix that had to be diagonalized for this value of $m$ had a dimension of 2,886 .

Though the calculations used to generate fig. 14 only targeted the ground state, they produced very accurate results for low-lying excited states as well. For $m=200$, the lowest three excitation energies were reproduced to $1 \%$ or better.

Reference 27] also reported results for a much larger problem, involving 40 particles in a $j=99 / 2$ orbit. In this case, the total number of $M=0$ states was $3.84 \times 10^{25}$, much too large for exact diagonalization. Here too the results were found to converge exponentially, and the estimated error for the ground state correlation energy at $m=100$ was just one part in $10^{4}$.

The first reported attempt at a realistic application of the phDMRG method in nuclear structure was presented in ref. [28. In that work, the nucleus ${ }^{24} M g$, with four neutrons and four protons outside doubly-magic ${ }^{16} O$, was studied. As is usual in the nuclear shell model, the $1 s$ and $1 p$ core orbits of ${ }^{16} O$ were assumed to be filled and inert 


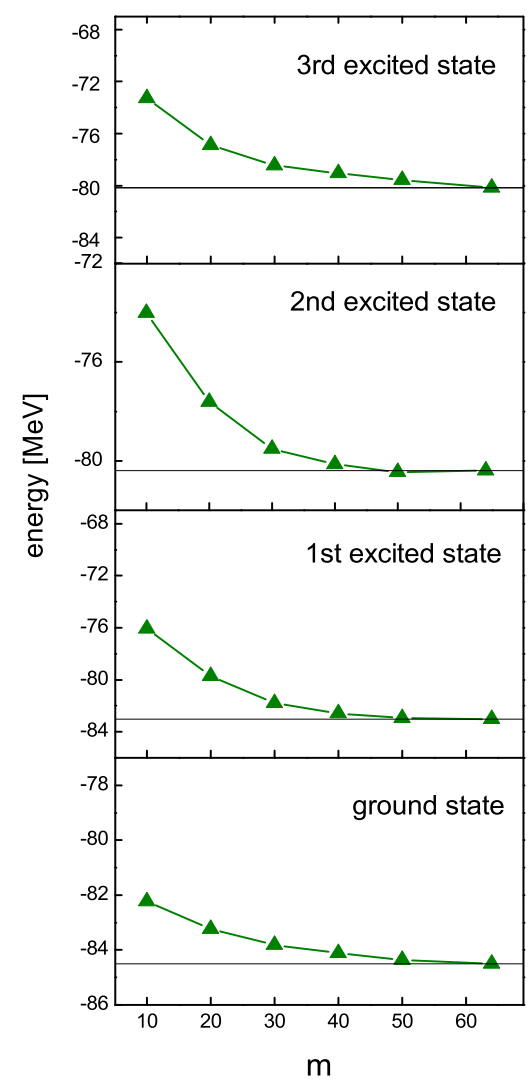

Figure 16. The calculated energies of the ground state and the three lowest excited states of ${ }^{24} \mathrm{Mg}$ as a function of $m$, the number of states retained in each block. The horizontal solid lines refer to the exact energies for the four states. Taken from ref. 28.

and the remaining 8 nucleons were restricted to the orbits of the $2 s-1 d$ shell. This shell-model problem is small and easily solved by exact shell-model diagonalization.

The results reported in ref. [28] were obtained using two different prescriptions for the ordering in which single-particle levels were included in the phDMRG algorithm. Here we show the results obtained using an axially-symmetric Hartree Fock prescription, with the active levels illustrated in fig. 15. The axially-symmetric HF single-particle levels are all doubly degenerate, thereby facilitating the use of the usual iterative DMRG scheme for adding levels. The calculations were done using the so-called USD effective Hamiltonian, which has been tailored to nuclei in the $2 s-1 d$ shell. Note that the DMRG method employed in these calculations is closely related to the two-grain DMRG reported in 69] and discussed in Sect. III.B.

Figure 16 shows the results for the energies of the lowest four states of the nucleus. For this problem the largest $m$ value that can be realized is $m=64$. The key point to note is that the convergence to the exact result (shown by the solid line) is very slow. A value of $m$ above 40 is required to get acceptably accurate reproduction of the exact results. Unfortunately, for such values of $m$, the matrices that must be treated 
are not much smaller than those of the full problem. Clearly, the phDMRG, method, as implemented in ref. [28], does not work very well for ${ }^{24} \mathrm{Mg}$.

There are several possible reasons for the failure of these calculations. One is that the infinite algorithm that was used cannot capture the correlations between the different single-particle levels in the problem. Unfortunately, it is difficult to incorporate sweeping in the phDMRG, especially for systems with active neutrons and protons, because of the preponderance of blocks involved. A second is that the method, as used, does not preserve rotational invariance. In the following section, we discuss how rotational invariance, and other non-Abelian symmetries, can be built into the DMRG. A third concerns the order in which the levels were included. As noted in sect. 3.1, a HF ordering, or indeed any ordering based solely on single-particle energy considerations, may not be optimal. Finally there are possible issues regarding implementation of the DMRG truncation algorithm. In particular, no effort was made in the calculation of ref. 28. to ensure that at least one state of each partition (number of particles/holes and their spin projection) was maintained in each iteration. In a more traditional DMRG study of ${ }^{24} \mathrm{Mg}$, it was found that implementation of such a condition dramatically improved convergence of the results [72].

\section{Restoration of Symmetries}

\subsection{General remarks}

Symmetries are a crucial element of all quantum systems. The invariance of a system under a group of symmetry transformations gives rise to conserved quantum numbers for the physical states of the system and associated degeneracies. In the case of uniform quantum lattices, translational invariance is a natural symmetry, with the associated conserved quantum number being the linear momentum. In spin lattices, the total spin of the system is likewise a conserved quantum number for all physical states. And in finite Fermi systems, total angular momentum is conserved as a reflection of the rotational invariance of the system.

Methods of approximately solving the Schrödinger equation frequently break the symmetries of the Hamiltonian and thereby lead to states that do not contain the appropriate quantum numbers. Often this is done intentionally, as spontaneous breaking of symmetries is a natural way to include collective correlations between the particles in the system. It is then critical, however, to restore the broken symmetries in order to establish contact with the states of the physical system, i.e., those probed in experiment.

The DMRG method, being a truncation of the full Hilbert space of the problem, in general violates symmetries, unless it is implemented very carefully. To understand this, consider the problem of a spin lattice, where we gradually add spin sites as we grow the system. To construct states of good total spin, it is necessary to include all the spin projections required by the Clebsch Gordan series. Truncation to a limited number of 
states based solely on density matrix considerations will not in general keep all the spin configurations needed to reconstruct the full spin eigenstates.

As pointed out by White [6], it is possible to preserve spin symmetry, in principle, by careful implementation of the density matrix strategy. In particular, as long as the density matrix truncation always includes all states from degenerate multiplets, it will preserve spin symmetry. However, because of the numerical limitations inherent in computational algorithms, it is extremely difficult to guarantee that we do not eliminate states that in a perfect calculation were degenerate with those that are kept. Equally important, it is not feasible to fix the number of states kept a priori in such a calculation, since that number is dictated by the sizes of the degenerate multiplets.

For the above reasons, several groups have been looking into alternative ways to conserve symmetries within the DMRG algorithm. In this section, we briefly review the important progress that has been made.

The first significant progress along these lines was the work of Sierra and Nishino 34. In their work, they showed that Hamiltonians with a continuous symmetry could be recast into the form of Interaction Round a Face (IRF) Hamiltonians, familiar in statistical mechanics. They then showed how to develop a DMRG algorithm appropriate to IRF Hamiltonians. The method was applied to several spin chains with impressive success. Examples include the spin-1/2 [34, spin-1 and spin-2 [35] Heisenberg chains and the XXZ chain 34. In all cases considered, the method achieved significantly greater accuracy than the usual DMRG algorithm when keeping the same number of states.

Despite the historical importance of the IRF-DMRG algorithm in the proper incorporation of symmetries in the DMRG, it is difficult to imagine its use for more complex systems with more complicated symmetries. Where major progress was made towards the development of a general framework for incorporating symmetries in the DMRG was through the work of McCulloch and Gulácsi [36. Their work presented a formalism that could be applied to any group of symmetry transformations, through proper construction of basis states that preserve the symmetry. They refer to this as the non-Abelian DMRG method. In the following subsection, we describe in some detail how their method can be applied to a finite Fermi system with conserved total angular momentum. We will refer to this specific example of their method as the jDMRG algorithm.

\subsection{Details on the jDMRG algorithm}

In htis subsection, we develop the jDMRG formalism, showing in some detail how it can be implemented for finite Fermi systems so as to produce exact angular momentum eigenstates. For simplicity, we restrict the discussion to systems with but one type of particle. For systems with two or more types of particles, atomic nuclei being an example, some slight modifications are required.

We begin by stating the problem. We wish to solve the Schrödinger equation for a 
set of $N$ identical particles subject to a Hamiltonian written in second-quantized form as

$$
H=-\sum_{j} \hat{j} \varepsilon_{j}\left(a_{j}^{\dagger} \widetilde{a}_{j}\right)_{0}^{0}+\sum_{j_{1} j_{2} j_{3} j_{4} L} X_{j_{1} j_{2} j_{3} j_{4}}^{L}\left[\left(a_{j_{1}}^{\dagger} a_{j_{2}}^{\dagger}\right)^{L}\left(\widetilde{a}_{j_{3}} \widetilde{a}_{j_{4}}\right)^{L}\right]_{0}^{0},
$$

where $\widehat{j}=\sqrt{2 j+1}$.

Note that we are assuming here that the particles under discussion are created (annihilated) in the single-particle state $\{j m\}$ by the operator $a_{j m}^{\dagger}\left(a_{j m}\right)$ and that the Irreducible Tensor Operator associated with the single-particle annihilation operator is defined by $\widetilde{a}_{j m}=(-)^{j-m} a_{j-m}$. By working in terms of the Irreducible Tensor Operators $a_{j m}^{\dagger}$ and $\widetilde{a}_{j m}$, we are able to couple operators directly and thus properly reflect the scalar nature of the Hamiltonian. Note that we use a coupling notation whereby $(\ldots)_{M}^{J}$ and $[\ldots]_{M}^{J}$ refer to coupled Irreducible Tensor Operators of rank $J$ and projection $M$.

The $N$ particles will be restricted to a finite set of single-particle levels $\{j m\}$, as depicted schematically in figure 17] for five orbits. The orbits are shown in ascending order according to their single-particle energies $\varepsilon_{j}$.

The jDMRG procedure involves three stages. The first is a preliminary stage, in which all necessary information on the individual active single-particle orbits are calculated and stored. The second is the "warm-up" stage, familiar to all DMRG algorithms. And the third is the likewise familiar "sweeping stage".

5.2.1. The preliminary stage. As the procedure will include the effects of all active single-particle levels in an angular-momentum coupled representation, we must store the following:

- All single-shell states $\left|j_{i}, n_{i}, \alpha_{i}, J_{i}\right\rangle$, for the various active orbits $\left\{j_{i}\right\}$. Here $n_{i}$ refers to the number of particles in the state and $\alpha$ refers to any additional quantum numbers needed to fully describe the state of total angular momentum $J_{i}$.

- The one-particle Coefficients of Fractional Parentage (CFPs) for all active orbits. These are the expansion coefficients required to express an antisymmetrized $n$ particle state as a product of antisymmetrized states of $n-1$ particles and a single particle.

- The reduced (or double-bar) matrix elements of all possibly relevant operators for those orbits, namely $a_{j}^{\dagger},\left[a_{j}^{\dagger} a_{j}^{\dagger}\right]^{K},\left[a_{j}^{\dagger} \tilde{a}_{j}\right]^{K},\left[\left\{a_{j}^{\dagger} a_{j}^{\dagger}\right\} \tilde{a}_{j}\right]^{K}$ and $\left[\left\{a_{j}^{\dagger} a_{j}^{\dagger}\right\}^{K}\left\{\tilde{a}_{j} \tilde{a}_{j}\right\}^{K}\right]^{0}$.

The reduced matrix elements of $\tilde{a}_{j}$ and $a_{j}^{\dagger}$ are very simply related to the one-particle CFPs. The reduced matrix elements of the more-complicated product operators can be obtained from them using well-known formulae [48.

5.2.2. The warm-up stage. We now discuss the warm-up phase, in which we build a first approximation to the dominant structure within groups of active orbits. As we will see, most of the formalism we develop for the warm-up phase will also be needed in the 


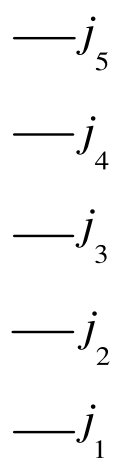

(a)

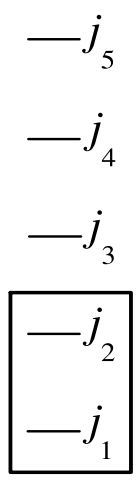

(b)

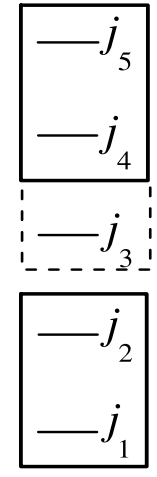

(c)

Figure 17. Schematic representation of the steps of the the jDMRG method.

sweep phase. In fig. 17b, we schematically illustrate the "warm-up" phase for the same set of five orbits.

We start off with the first orbit $j_{1}$, for which all the single-shell matrix elements were calculated in the "preliminary" stage. We then add the second orbit, forming an enlarged "block". This is schematically illustrated in fig. 17b, where the enlarged block contains orbits $j_{1}$ and $j_{2}$.

Next, we must build the reduced matrix elements of all relevant sub-operators of the Hamiltonian for the enlarged block. To see how this is done, we consider a generic block involving $k$ orbits, $\left|j_{1} \cdots j_{k}, n, \alpha, J\right\rangle$. The enlarged block basis, in which orbit $j_{k+1}$ has been added, is $\left|j_{1} \cdots j_{k}, n, \alpha, K ; j_{k+1}, m, \beta, L(J)\right\rangle$. The matrix elements of the single-particle creation operator $a_{i}^{\dagger}$ in the enlarged block are

$$
\begin{aligned}
& \left\langle j_{1} \ldots j_{k}, n^{\prime}, \alpha^{\prime}, K^{\prime} ; j_{k+1}, m^{\prime}, \beta^{\prime}, L^{\prime}\left(J^{\prime}\right)\left\|a_{i}^{\dagger}\right\| j_{1} \ldots j_{k}, n, \alpha, K ; j_{k+1}, m, \beta, L(J)\right\rangle \\
& =\delta_{\beta \beta^{\prime}} \delta_{m m^{\prime}} \delta_{L L^{\prime}}(-)^{K^{\prime}+L^{\prime}+J+i} \widehat{J} \widehat{J}^{\prime}\left\{\begin{array}{ccc}
K^{\prime} & J^{\prime} & L^{\prime} \\
J & K & i
\end{array}\right\} \\
& \quad \times\left\langle j_{1} \cdots j_{k}, n^{\prime}, \alpha^{\prime}, K^{\prime}\left\|a_{i}^{\dagger}\right\| j_{1} \cdots j_{k}, n, \alpha, K\right\rangle+ \\
& \quad+\delta_{\alpha \alpha^{\prime}} \delta_{n n^{\prime}} \delta_{K K^{\prime}}(-)^{K^{\prime}+L+J^{\prime}+i} \widehat{J^{\prime}} \widehat{J}^{\prime}\left\{\begin{array}{ccc}
L^{\prime} & J^{\prime} & K^{\prime} \\
J & L & i
\end{array}\right\} \\
& \quad \times\left\langle j_{k+1}, m^{\prime}, \beta^{\prime}, L^{\prime}\left\|a_{i}^{\dagger}\right\| j_{k+1}, m, \beta, L\right\rangle .
\end{aligned}
$$

It involves a reduced matrix element from the previous iteration (which has already been calculated) and a reduced matrix element for the orbit that is being added (which was calculated in the preliminary stage). Thus, all information is available to calculate this reduced matrix element, and indeed all others, in the enlarged block.

As in all DMRG algorithms, we now wish to choose out of all the states in the enlarged block those to keep. Here the strategy is somewhat different depending on whether there is only one kind of particle or two. If there are two kinds of particle, 
one can couple the corresponding states together, build and diagonalize the superblock Hamiltonian and then truncate in the sector of interest by using its reduced density matrix, assuming each type of particle is the medium for the other.

When there is only one kind of particle, as in the example under discussion, an alternative strategy must be found. A natural one is to use the Wilson RG strategy of diagonalizing the Hamiltonian in the enlarged space and keeping the $m$ lowest eigenstates for each value of $n$ and $J$.

Following truncation, the reduced matrix elements for the truncated enlarged block are calculated and stored on hard disk. This is done for all size blocks, $j_{1}, j_{1} \rightarrow j_{2}$, $j_{1} \rightarrow j_{3}, j_{1} \rightarrow j_{4}$, and $j_{1} \rightarrow j_{5}$.

5.2.3. The sweep stage. We now discuss the sweep stage, whereby we sweep back and forth through the single-particle levels to improve the description of each block by optimally taking into account its correlation with all other levels.

The sweep stage is schematically illustrated in fig. 17k for the same five orbits. At the point represented by the figure, we are sweeping backwards through the singleparticle levels and have treated as a block the last two levels, $j_{5}$ and $j_{4}$. We wish to enlarge this block to include level $j_{3}$. The medium for this enlarged will be levels $j_{1} \rightarrow j_{2}$.

The sweep stage involves first a calculation of all relevant matrix elements in the enlarged block consisting of levels $j_{3} \rightarrow j_{5}$. This can be done using exactly the same formulae developed for the warm-up stage, with one example given in Eq. (32).

Once we have calculated all matrix elements in the enlarged block, we construct the states of the superblock by coupling those of the enlarged block (orbits $j_{3} \rightarrow j_{5}$ ) to those of the medium (orbits $j_{1} \rightarrow j_{2}$ ), viz: $\left|j_{1} \cdots j_{2}, n, \alpha, K ; j_{3} \cdots j_{5}, m, \beta, L(J)\right\rangle$. We only construct states with the correct total number of particles $N=n+m$.

The calculation of the Hamiltonian matrix in the superblock involves a sum of terms, each being a product of a reduced matrix element in the enlarged block (just calculated) and a reduced matrix element in the medium (output from the previous sweep or the warm up).

As always, the sweep stage is iterated until acceptable convergence has been achieved.

\subsection{Applications of the non-Abelian DMRG formalism}

The first application [36] of the non-Abelian DMRG method was reported by McCulloch and Gulácsi for the one-dimensional Hubbard model. This model has the usual spin symmetry and also a pseudo-spin symmetry generated by the set of operators $I^{+}=\sum_{i}(-)^{i} c_{i \uparrow}^{\dagger} c_{i \downarrow}^{\dagger}, I^{-}=\sum_{i}(-)^{i} c_{i \downarrow} c_{i \uparrow}$, and $I^{z}=\sum_{i} \frac{1}{2}\left(n_{i \uparrow}+n_{i \downarrow}-1\right)$. Thus the DMRG algorithm can be implemented at three levels: (1) by only including the spin projection as a conserved quantum number (called $U(1)$ ), (2) by including the total spin as a conserved quantum number (called $S U(2)$ ) and (3) by considering both the total spin and the pseudo-spin as good quantum numbers (called $S O(4)$ ). There is also an additive 
Table 5. Comparison of results for three sets of DMRG calculations for the ground state of the half-filled 60-site Hubbard model with $t=U=1$. The CPU time is in seconds per sweep. (1) $U(1) \times U(1)$ refers to a calculation in which number conservation and conservation of spin projection were imposed; (2) $U(1) \times S U(2)$ also includes total spin conservation; (3) $S O(4)$ in addition includes pseudo-spin conservation. Extracted from Table I of ref. [36].

\begin{tabular}{cccccr}
\hline Basis & $m$ & $D$ & $E$ & $\left(E-E_{g}\right) /\left|E_{g}\right|$ & $\mathrm{CPU}$ \\
\hline$U(1) \times U(1)$ & 100 & 100 & -76.7484986435 & $4.2 \times 10^{-5}$ & 10 \\
$U(1) \times U(1)$ & 300 & 300 & -76.7516910404 & $6.3 \times 10^{-7}$ & 110 \\
$U(1) \times S U(2)$ & 100 & 226 & -76.7515581914 & $2.3 \times 10^{-6}$ & 15 \\
$U(1) \times S U(2)$ & 300 & 716 & -76.7517389831 & $1.1 \times 10^{-8}$ & 158 \\
$S O(4)$ & 100 & 526 & -76.7517351742 & $6.1 \times 10^{-8}$ & 18 \\
$S O(4)$ & 300 & 1766 & -76.7517398448 & $7.9 \times 10^{-11}$ & 133 \\
\hline
\end{tabular}

(or Abelian) symmetry of number conservation (likewise $U(1)$ ), which can be included at each of the three levels. Results for the three levels of DMRG application are summarized in table 5 for a half-filled 60-site Hubbard lattice with $t=U=1$.

The table includes a quantity $D$, which in the case of a non-Abelian symmetry is equal to the number of states in the Abelian analysis required to get the same results as in the symmetry-adapted analysis. Thus, for example, a calculation in which the full $\mathrm{SO}(4)$ symmetry is preserved and in which a total of $\mathrm{m}=100$ states are maintained in a block would require $m=526$ states for comparable accuracy when only the z-projection and the particle number are conserved. The table also indicates the computational effort required for these various calculations. Keeping the same number of states in a calculation with full symmetry restoration as in a calculation with only additive symmetries increases the cost in CPU time very little, but yields an enormous improvement in accuracy.

The results shown in Table 5 are for the the $\mathrm{S}=0$ ground state of the system. Calculations of higher spin states do not lead to as dramatic an improvement and cost savings.

Subsequently, the method was applied to the two-dimensional t-J Model for lattices up to $24 \times 6$ [37. Here the purpose was no longer to simply assess the usefulness of symmetry restoration in the DMRG approach, but rather to use the power of the method to provide useful insight into this model of possible relevance to high- $T_{c}$ superconductors. In particular, it attempted to address the important, but controversial, issue of stripe formation in the t-J model.

The analysis made use of the Liang and Pang approach to 2D lattices [4], which unrolls the 2D lattice onto a $1 \mathrm{D}$ lattice with long-range interactions. The calculations included up to $m=1200$ states per block. An indication of the accuracy of the method is given in Table 6, where the results obtained for a $16 \times 6$ lattice with full spin conservation are compared with those having conserved spin projection only [50]. As can be readily seen, the calculations with full $\mathrm{SU}(2)$ spin conservation lead to significantly lower 
Table 6. Comparison of results of DMRG calculations for the ground state of a $16 \times 6$ t-J system with $J=0.35, t=1$, eight holes and cylindrical boundary conditions. The results labelled U(1) only preserve spin projection and are from ref. [50. Those labelled SU(2) involve full spin conservation. An extrapolated "true" ground state energy is also shown. From Table I of ref. 37]. Reprinted by permission of Taylor \& Francis Ltd., http://www.tandf.co.uk/journals/titles/14786435.

\begin{tabular}{ccc}
\hline Basis & $m$ & $E$ \\
\hline$U(1)$ & 1000 & -52.279 \\
$S U(2)$ & 500 & -52.284 \\
$S U(2)$ & 800 & -52.463 \\
$S U(2)$ & 1200 & -52.520 \\
- & $\infty$ & $-52.65 \pm 0.05$ \\
\hline
\end{tabular}

energies for comparable block sizes. The ground state emerges from this calculation in a two-stripe configuration, in agreement with ref. [50].

More recently, the method was applied to the problem of localized spin ordering in Kondo lattice models [73. By exactly preserving spin symmetry in the problem, the authors were able to directly measure the magnetization of the ground state and this made it possible for them to confirm the existence of a second ferromagnetic phase in the model [74, for which weak signals had been observed in a previous DMRG study that did not fully restore symmetries [75].

\section{Summary and Outlook}

In this review, we have described the current status of efforts to develop the Density Matrix Renormalization Group for application to finite Fermi system. Enormous progress has been made on this front and the method has indeed been applied with impressive success in several areas, especially in applications to Quantum Chemistry, to study the physics of ultrasmall superconducting grains and to study the properties of two-dimensional electron systems. Along with these applications has come much work aimed at identifying the key issues that must be resolved in order to build an optimized DMRG algorithm for use in these and other systems. One of the most important issues concerns the optimal approach to ordering the single-particle basis states that are iteratively added by the DMRG algorithm. While important progress has been made, questions still remain and more work remains to be done.

Another area in which important progress has been made concerns the full incorporation of symmetries in the DMRG method. It is now possible to consistently incorporate non-Abelian symmetries, such as rotational invariance, in addition to additive or Abelian symmetries. Early applications of the associated new methodology suggest that it permits increased accuracy in DMRG calculations, while requiring less CPU cost. More work along these lines is clearly warranted. 
Another interesting point that has recently emerged concerns the usefulness of the DMRG method when treating weakly-coupled strongly-correlated subsystems. An application of the particle-hole DMRG method to tunnelling between ultrasmall superconducting grains suggests that the methodology breaks down when the tunnel coupling between the two grains is very weak. Similar issues also arise when dealing with a set of single-particle levels that involve well-separated subsets (e.g. shells), which are also very weakly coupled [72]. It would be interesting to see whether the DMRG algorithm can be appropriately modified to handle such scenarios. A possibility might be to apply the DMRG separately to the various subsystems and then to couple them perturbatively.

The issue of the ordering of levels for their optimal treatment within the DMRG method has recently led to the suggestion that Quantum Information Theory might provide a useful handle on the problem. This connection needs further exploration.

We have focussed our remarks on some of the most critical questions that have recently been raised in the context of the DMRG method as it applies to finite Fermi systems and some of the most interesting methodological breakthroughs. As further progress is made to address these and other issues regarding the optimal protocol for DMRG studies, we expect a rapid increase in the number of applications. A potentially fertile area that has not yet been explored is the atomic nucleus.

In closing, we believe that the Density Matrix Renormalization Group method will continue to grow as a viable method for carrying out reliable and extremely accurate calculations for the properties of complex finite Fermi systems, adding to the enormous history of success it has already achieved in the description of quantum lattices.

\section{Acknowledgments}

We would like to express our appreciation to Mario Stoitsov, Sevdalina Dimitrova, German Sierra, Nicu Sandulescu and Larisa Pacarescu, all of whom made important contributions to the DMRG projects on which we have worked. Helpful discussions with Willy Dussel, David Dean and especially Thomas Papenbrock on aspects of the DMRG method are also gratefully acknowledged. This work was supported in part by the Spanish DGI under grant BFM2003-05316-C02-02 and in part by the US National Science Foundation under grant \#s PHY-9970749 and PHY-0140036.

\section{References}

[1] Wilson K G 1975 Rev. Mod. Phys. 47773.

[2] Kondo J 1964 Prog. Theor. Phys. 3237.

[3] White S R 1992 Phys. Rev. Lett. 692863.

[4] White S R 1993 Phys. Rev. B 4810345.

[5] White S R and Huse D A 1993 Phys. Rev. B 483844.

[6] White S R 1998 Phys. Rep. 301187.

[7] Hallberg K A, Horsch P and Martinez G 1995 Phys. Rev. B 52 R719. 
[8] White S R, Noak R M and Scalapino D J 1994 Phys. Rev. Lett. 73 886; White S R 1999 Phys. Rev. B 5352.

[9] White S R and Affleck I 1996 Phys. Rev. B 549862.

[10] Chen L and Moukouri S 1996 Phys. Rev. B 531866.

[11] White S R, Affleck I, and Scalapino D J 2002 Phys. Rev. B 65 165122; Ramasesha S, Pati S K, Krishnamurthy H R, Shuai Z, and Brédas J L 1996 Phys. Rev. B 54, 7598 (1996).

[12] Roth R and Schollwöck U 1998 Phys. Rev. B 589264.

[13] Mikeska H-J, Neugebauer U, and Schollwöck U 1997 Phys. Rev. B 552955.

[14] Lepetit M-B and Pastor G M 1997 Phys. Rev. B 56 4447; Boman M and Bursill R J 1998 Phys. Rev. B 5715167.

[15] Shibata N and Ueda K 1999 J. Phys.: Condens. Matter 11 R1.

[16] White S R 1996 Phys. Rev. Lett. 773633.

[17] White S R and Scalapino D J 1997 Phys. Rev. B 556504.

[18] Peschel I, Wang X, Kaulke M and Hallberg K (Eds.) 1999 Density Matrix Renormalization: A New Numerical Method in Physics, Lectures Notes in Physics (Berlin, Springer).

[19] Xiang T 1996 Phys. Rev. B 5310445.

[20] White S R and Martin R L 1999 J. Chem. Phys. 1104127.

[21] Daul S, Ciofini I, Daul C and White S R 2000 Int. J. Quantum Chem. 79331.

[22] Mitrushenko A O, Fano G, Ortolani F, Linguerri R and Palmeri P 2001 J. Chem. Phys. 1156815.

[23] Chan G K-L and Head-Gordon M 2002 J. Chem. Phys. 1164462.

[24] Dukelsky J and Sierra G 1999 Phys. Rev. Lett. 83172.

[25] Dukelsky J and Sierra G 2000 Phys. Rev. B 6112302.

[26] Dukelsky J and Pittel S 2001 Phys. Rev. C 63 R061303.

[27] Dukelsky J, Pittel S, Dimitrova S S and Stoitsov M V 2002 Phys. Rev. C 65054319.

[28] Pittel S, Dukelsky J, Dimitrova S and Stoitsov M 2003 Rev. Mex. Física 49, Suppl. 482.

[29] Shibata N and Yoshioka D 2001 Phys. Rev. Lett. 865755.

[30] Yoshioka D and Shibata N 2002 Physica E12 43.

[31] Shibata N and Yoshioka D 2003 J. Phys. Soc. Japan 72664.

[32] Shibata N 2003 J. Phys. A: Math. Gen. 36 R381.

[33] Shibata N and Yoshioka D 2003 Preprint cond-mat/0308122

[34] Sierra G and Nishino T 1997 Nucl. Phys. B 495505.

[35] Wada T 2000 Phys. Rev. E 613199.

[36] McCulloch I P and Gulácsi M 2002 Europhys. Lett. 57852.

[37] McCulloch I P, Bishop A R and Gulacsi M 2001 Phil. Mag. B81 1603.

[38] Cramer C J 2003 Essentials of Computational Chemistry: Theories and Models (Chichester, John Wiley and Sons).

[39] Ring P and Peter Schuck P 1980 The Nuclear Many-Body Problem (New York, Springer-Verlag).

[40] Hubbard J 1963 Proc. R. Soc. A276 238; -1978 Phys. Rev. B 17494.

[41] Heyde K 1990 The Nuclear Shell Model (Berlin, Springer-Verlag).

[42] Chui S-T and Bray J W 1978 Phys. Rev. 18 2426; Bray J W and Chui S-T 1979 Phys. Rev. 19 4876.

[43] Anderson P W 1961 Phys. Rev. 1641.

[44] Golub G H and Van Loan C F 1989 Matrix Computations, Second Edition, (Baltimore and London, Johns Hopkins Press).

[45] Papenbrock T and Dean D J 2003 Phys. Rev. C 67051303.

[46] Nishimoto S, Jeckelmann E, Gebhard F and Noack R M 2002 Phys. Rev. B 65165114.

[47] White S R and Scalapino D J 2003 Preprint cond-mat/0306545

[48] De-Shalit A and Talmi I 1963 Nuclear Shell Theory, (New York and London, Academic Press).

[49] Liang S and Pang H 1994 Phys. Rev. B 499214.

[50] White S R and Scalapino D J 1999 Preprint cond-mat/9907243.

[51] Legeza O, Roder J and Hess B A 2003 Phys. Rev. B 67125114. 
[52] Legeza O and Solyom J 2003 Preprint cond-mat/0305336.

[53] Anusooya Y, Pati S K, Ramasesha S 1997 J. Chem. Phys. 1061.

[54] Yaron D, Moore E E, Shuai Z, and Brédas J L 1998 J. Chem. Phys. 1087451.

[55] Fano G, Ortolani F and Ziosi L 1998 J. Chem. Phys. 108, 9246.

[56] Bendazzoli L, Evangelisti S, Fano G, Ortolani F and Ziosi L 1999 J. Chem. Phys. 1091277.

[57] Bursill R J and Barford W 1999 Phys. Rev. Lett. 821514.

[58] Barford W, Bursill R J and Lavrentiev M Y 2001 Phys. Rev. B 63195108.

[59] Race A, Barford W and Bursill R J 2001 Phys. Rev. B 6435208.

[60] Bursill R J and Barford W 2002 Phys. Rev. B 66205112.

[61] Race A, Barford W and Bursill R J 2003 Phys. Rev. B 67245202.

[62] Anderson P W 1959 J. Phys. Chem. Solids 1128.

[63] Ralph D C, Black C T and Tinkham M 1996 Phys. Rev. Lett. 76 688;_1997 Phys. Rev. Lett. 784087.

[64] Von Delft J et al. 1996 Phys. Rev. Lett. 773189.

[65] Braun F and Von Delft J 1998 Phys. Rev. B 599527.

[66] Mastellone A, Falci G and Fazio R 1998 Phys. Rev. Lett. 804542.

[67] Braun F and von Delft J 1998 Phys. Rev. Lett. 814712.

[68] Richardson R W 1966 Phys. Rev. 141949.

[69] Gobert D, Schollwöck U and Von Delft J 2003 Preprint cond-mat/0305361.

[70] Stone M, Wyld H W, and Schult R L 1992 Phys. Rev. B 4514156.

[71] Mitra S and A.H. MacDonald A H 1993 Phys. Rev. B 482005.

[72] Papenbrock T and Dean D J 2003 Private communication.

[73] McCulloch I P, Juozapavicius A, Rosengren A and Gulacsi M 2002 Phys. Rev. B 65052410.

[74] Tsunetsugu H, Sigrist M and Ueda K 1993 Phys. Rev. B 478345.

[75] Shibata N, Ueda K, Nishino T and Ishii C 1996 Phys. Rev. B 5413495. 